Canadian University Music Review

Canadian University Music Review

Revue de musique des universités canadiennes

\title{
Perceptual Aspects of Tuning in a Balinese Gamelan Angklung for North American Students
}

\section{Jay Rahn}

Volume 16, numéro 2, 1996

URI : https://id.erudit.org/iderudit/1014424ar

DOI : https://doi.org/10.7202/1014424ar

Aller au sommaire du numéro

\section{Éditeur(s)}

Canadian University Music Society / Société de musique des universités canadiennes

\section{ISSN}

0710-0353 (imprimé)

2291-2436 (numérique)

Découvrir la revue

Citer cet article

Rahn, J. (1996). Perceptual Aspects of Tuning in a Balinese Gamelan Angklung for North American Students. Canadian University Music Review / Revue de musique des universités canadiennes, 16(2), 1-43.

https://doi.org/10.7202/1014424ar
Résumé de l'article

A detailed analysis of the spectra and frequencies of tones produced on metallophones in a Balinese orchestra clarifies a number of issues that arise in studying timbre and tuning. Among these are the relative importance of accuracy and completeness of measurements, the relevance of acoustical description to musical perception and style, the appropriateness of statistical generalizations and numerical formulations to musical practice and theory, and the possibility of bi-musical responses to divergent tuning systems. Byproducts of the investigation include a proposed basis for inter-cultural ear training and a novel formulation of the unison, octave, and other intervals (including wide and narrow variants), as well as traditional scales and interval collections that have existed side-by-side in widely varying forms within individual cultures.
All Rights Reserved (C Canadian University Music Society / Société de musique des universités canadiennes, 1996
Ce document est protégé par la loi sur le droit d'auteur. L’utilisation des services d'Érudit (y compris la reproduction) est assujettie à sa politique d'utilisation que vous pouvez consulter en ligne.

https://apropos.erudit.org/fr/usagers/politique-dutilisation/ 


\title{
PERCEPTUAL ASPECTS OF TUNING IN A BALINESE GAMELAN ANGKLUNG FOR NORTH AMERICAN STUDENTS
}

\author{
Jay Rahn
}

The goal of the present study is to provide an interpretation of tuning measurements made on Indonesian instruments, specifically, an interpretation that takes into account well-established findings in music psychology, stylistic observations and native testimony in ethnomusicology, and current formulations in music theory. ${ }^{1}$ As a byproduct of this study, I súggest ways in which contemporary musicianship training might assimilate aspects of such tunings. The central issue of the study is how one might best distinguish among various kinds and amounts of variation in the sizes of intervals within a musical tradition that features great diversity in its handling of frequencies. The resolution of this issue presented below results in another byproduct of the investigation, namely, a clarification of certain distinctions between pitch intervals and timbre. This clarification leads to the substitution of i) a paradigm based on psychoacoustics and perceived, essentially non-numerical relations for ii) the paradigm of frequency ratios that has undergirded important theories of music in various cultures for at least 2500 years.

This report focuses on the tunings of keyed metallophones and other fixed-frequency instruments employed traditionally in Indonesia. Such instruments have formed the cores of several kinds of ensemble (gamelan) in Indonesia and, by transplantation, the West. The tones of the keyed metallophones considered here are produced by striking individual slabs of metal. These keys vary extremely little in frequency once they have been tuned and

11 wish to thank Professor Ron Chandler of the Faculty of Music, University of Toronto, who generously made available to me the Faculty's Stroboconn for the tone measurements reported here. John Holm, a recent graduate of York University's Music programme, provided technical assistance in the spectral analysis, which was carried out in the Faculty of Fine Arts Interdisciplinary Computer Centre and in the Music Computer Lab of Winters College, both at York. These facilities were kindly made available to me by their directors, respectively, Keith Bradley and Michael Coghlan. Casey Sokol of York's Music Department also made available his equally tempered gender quartet for preliminary portions of this investigation (mentioned only briefly here). Max Brandt of Semester at Sea, University of Pittsburgh, graciously supplied fullsome documentation on the background of the particular ensemble that forms the focus of this study. Michael Wright of State College of New York, Buffalo, Ellen Koskoff of Eastman School of Music, Rochester, and Annette Sanger, of Toronto's University Settlement House, provided suggestive leads on interpreting connections between tuning and performance practice; nonetheless, I assume responsibility for any flaws in the conclusions reported here. The project as a whole was supported by two SSHRCC Internal Grants ("Analysis of Gamelan Tunings" and "Melodic Interval Perception by Western and non-Western Musicians") as well as a Specific Research Grant ("Elementary Tonal Structures"), all of these from York University. 
their metal has "set." The same situation holds for several non-keyed metal instruments of Indonesia, for example, various gongs. Measurements of individual frequencies produced by such Indonesian instruments can be valid for a very long time. Nonetheless, problems might arise in interpreting such measurements, especially as they have been reported and discussed in the quite extensive ethnomusicological literature on Indonesian tunings.

\section{Problems in Assessing Frequency Measurements of Indonesian Instruments}

Alexander Ellis, considered widely to have been the founder of ethnomusicology, used tuning forks to measure frequencies on Indonesian instruments. ${ }^{2}$ However, he noted that these were only accurate within about four cycles per second (cps or $\mathrm{Hz}$ ). ${ }^{3}$ For certain sorts of distinctions considered in this study, such a "span of tolerance," or range of measurement error, is far too wide.

Since Ellis's time, researchers have tended increasingly to employ much more accurate measuring devices, in particular, the Stroboconn, which, in principle, can ensure accuracy within a single cycle per second and a single cent (i.e., a hundredth of a semitone). However, quite recently, ethnomusicologist Roger Vetter, citing musicologist Albrecht Schneider, has suggested that even data based on Stroboconn measurements can be misleading, because such precise measurements do not acknowledge wide dispersions of frequency within individual tones. ${ }^{4}$ Nonetheless, I show below that such dispersion need not be regarded as being quite as problematic as Vetter's report might lead one to believe. Indeed, the majority of published figures for Indonesian tunings can be taken pretty much at face value, with allowances for measurements that have used such relatively inaccurate devices as Ellis's tuning forks.

Another possible source of difficulty consists in the fact that often frequency measurements of gamelan tones have been incomplete in ways highly relevant to the various styles of music traditionally played on the instruments. Early publications report the frequencies of tones produced only on a single instrument of an ensemble and solely within a single octave. In Indonesian tradition, individual tones within an octave are given distinct names (e.g., ding, dong, deng, dung, and dang in the solmization system of Bali). Both in Bali and elsewhere in Indonesia (e.g., where such terms as bem, gulu, dada, lima, and nem are used) note-names are repeated at the octave thoughout all registers, just as one finds in various systems of solmization-syllables, letter-names, numerals, and characters used in the West, the Middle East, South Asia, and East Asia.

2Alexander J. Ellis, "Tonometrical Observations on Some Existing Non-Harmonic Scales," Proceedings of the Royal Society 37 (1884): 368-85; cf. also his "Additions by the Translator," in Hermann L.F. Helmholtz, On the Sensations of Tone as a Physiological Basis for the Theory of Music, (2nd English ed., 1885; repr. New York: Dover, 1954): 430-556, esp. 518.

3 Ellis, "Additions by the Translator," 446.

4Roger Vetter, "A Retrospect on a Century of Gamelan Tone Measurements," Ethnomusicology 33, no. 2 (1989): 217-27, esp. n. 3, 219. 
Research during the past three decades 5 has shown that Balinese unisons are generally "out-of-tune." Two tones in the same register which are both called, for example, "ding" often differ in frequency by several cycles per second. In Bali, such "mis-tuning" is a result of deliberate efforts to create beating between tones. Both in Bali and elsewhere in Indonesia, tones of the same name (e.g., "ding," again) in adjacent registers, that is, an octave apart, differ in varying amounts from the frequency ratio $2 / 1$ ( $=1200$ cents) that has been regarded as an ideal of tuning for the octave in the West and other cultures. Accordingly, studies that report tunings only for a single instrument or register can be misleading. Nevertheless, the present study provides a basis for believing that even the rather fragmentary accounts found in some previous reports can be valuable in certain respects. Observed variations in unison- and octave-sizes are quite constrained and regular, and important interval relations exist between tones within any octave.

An additional source of concern is the extent to which acoustical descriptions might be relevant to the actual perception or practice of music traditionally played on the instruments. It is a truism of music psychology that acoustically measured differences in frequency are not uniformly significant to music as it is actually heard or performed. Some differences are quite slight or involve parts of a piece or texture that are rather remote, whereas others are much larger or more immediate. Earlier studies overlook such distinctions by treating all differences as though they were the same in importance. By contrast, the present report shows how a hierarchy of difference can be defined in a way closely connected with procedures and forms used in the music itself.

In the particular Indonesian genre considered in detail here, individual keyed metallophones are grouped two, four, eight, and ten at a time and constitute the core of an orchestra. In actual pieces, these instruments most often form unisons and octaves with each other. The kind of ensemble focused on in the present study, namely, Balinese gamelan angklung, features almost exclusively unisons and octaves as the vertical intervals between keyed metallophone tones struck simultaneously (Ex. 1). Among these core instruments, other intervals (e.g., "fifths," "fourths," etc.) arise for the most part, between tones that are attacked at different times, or that are sounded only by the uppermost instruments in the most complex, interlocking, "hocket" (kotekan) sections of the most intricate traditional pieces (Ex. 2).

Within Indonesian culture, entire ensembles of metallophones and other instruments have traditionally been played upon over periods of many years in the context of court life and village music clubs. Hardja Susilo has stressed that such ensembles are recognized as having distinct sonic personalities by virtue of their particular tunings. ${ }^{6}$ Mantle Hood has emphasized that individual orchestras have been accorded such proper names as "Venerable Dark Cloud" and "New Flower" because of their idiosyncratic interval patterns. ${ }^{7}$ Capturing

5E.g., Mantle Hood, "Sléndro and Pélog Redefined," Selected Reports 1, no. 1 (1966): 28-48.

6 Hardja Susilo, review of Jaap Kunst, Music in Java, 3rd ed., in Asian Music 7, no. 1 (1975): 58-68.

7Hood, "Sléndro and Pélog Redefined." Cf. also Judith Becker, "Some Thoughts about Pather. Javanese Modal Classifications," paper presented to International Musicological Society, Berkeley (1977), as cited in W. Jay Dowling and Dane L. Harwood, Music Cognition (Orlando: Academic Press, Inc., 1986), 120. 

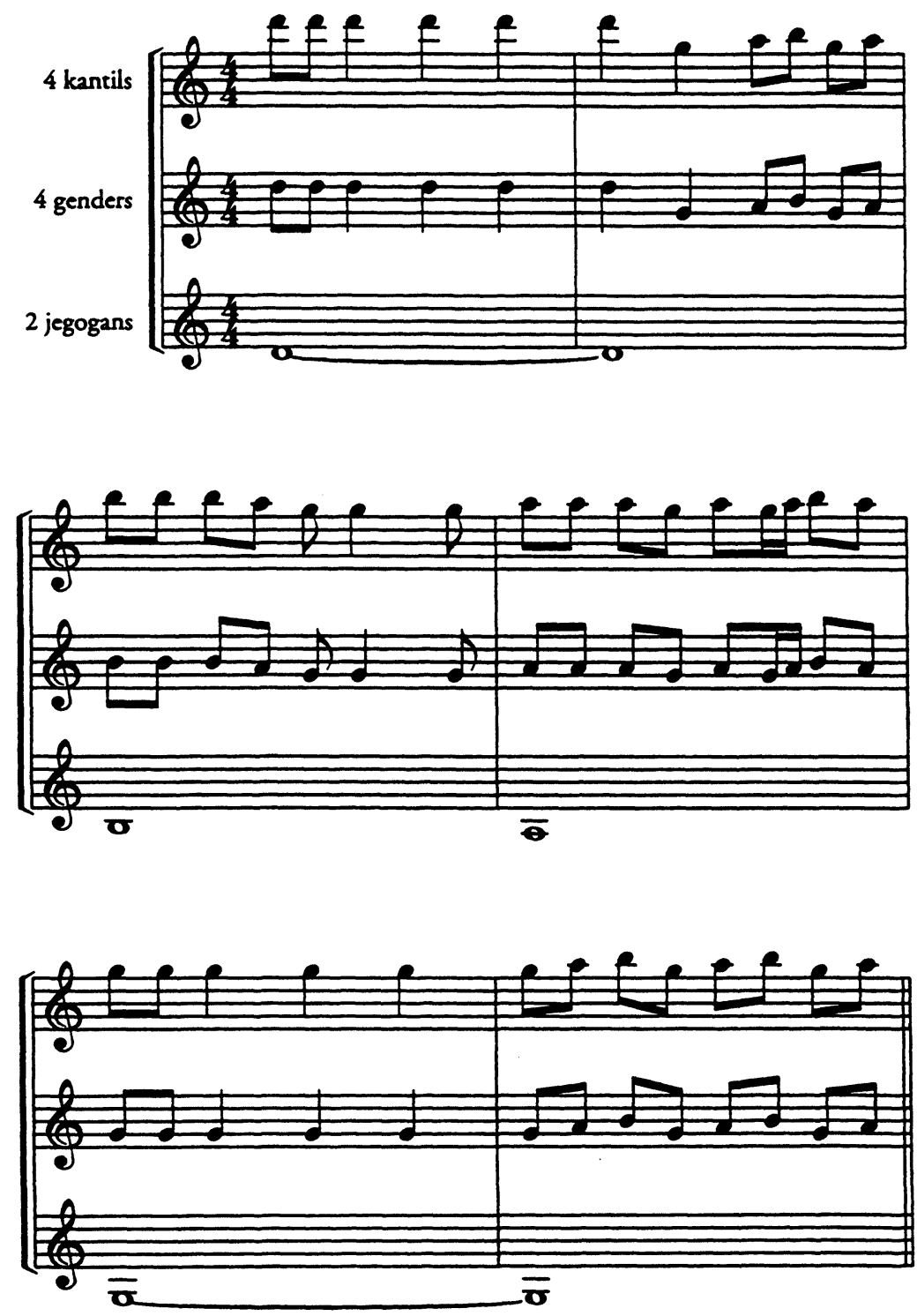

Example 1: Opening measures of "Jaran Sirig" (Rearing Horse), traditional piece for Balinese angklung ensemble (after McPhee, 1966; used with permission of Yale University Press). Noteworthy is the frequent use of simultaneous unisons, octaves, and fifteenths between instruments in the "normal" or "usual" style of angklung performance. 


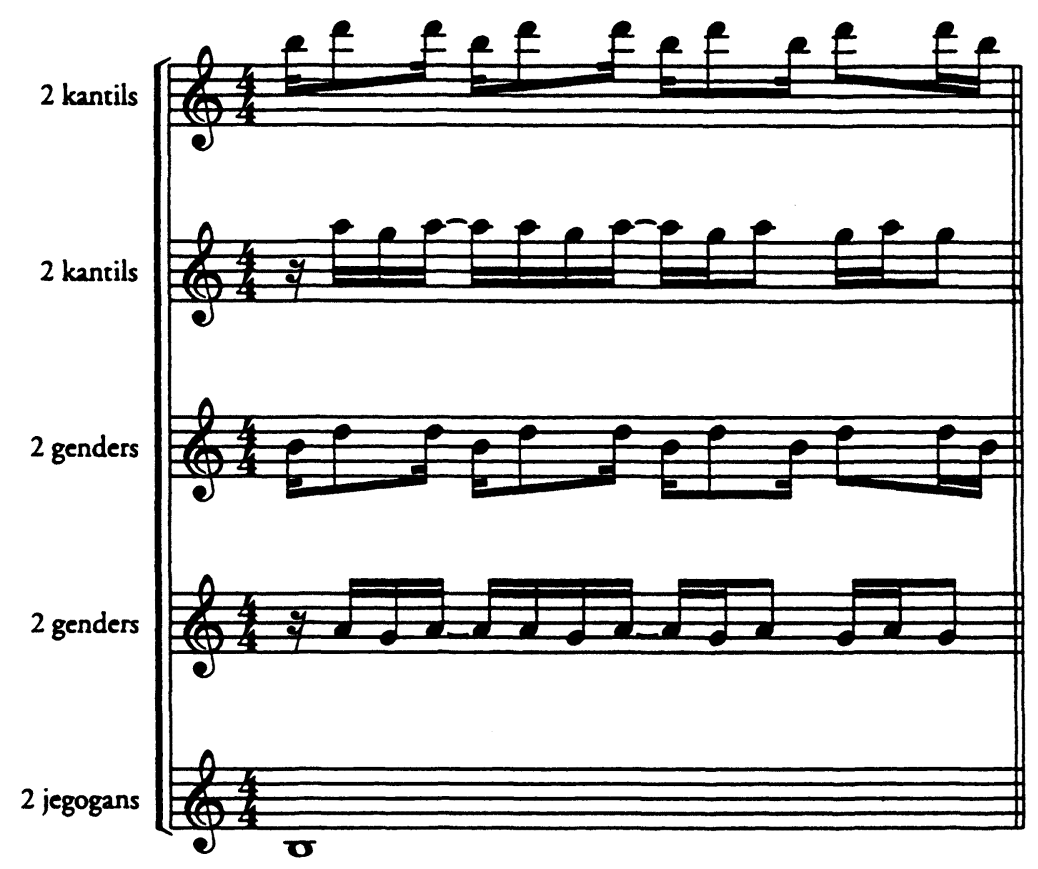

Example 2: Beginning of kotekan section of "Jaran Sirig" (see Example 1, above. After McPhee, 1966; used with permission of Yale University Press). Of particular importance is the relatively frequent use of simultaneous "fifths," "fourths," etc. in this more complex, "hocket" style.

aspects of such personalities requires distinguishing among the diverse musical roles that unisons, octaves, and other intervals play in creating an orchestra's sonic character.

Also of relevance are tendencies that are universal in human hearing. In studies of tuning and tonality, the findings in music psychology on which the present account rests have been either ignored or underexploited for so long that I generally reverse the usual practice in music psychology studies by citing early well-known accounts rather than the most recent. Recent psychological studies have not necessitated substantial revisions of the topics treated here. Moreover, citing early, widely disseminated studies highlights the fact that all the ingredients of a substantial paradigm shift in studies of tuning have existed both earlier than, and alongside, various investigations they meanwhile have failed to affect.

Each of the ten metallophones considered intensively in this study comprises four slabs of metal. The forty tones that result might seem relatively few in comparison with, say, the eighty-eight tones of a Western piano. However, it should be emphasized that the forty metallophone tones of the angklung ensemble examined here give rise to almost 800 pairs of tones, that 
is, intervals $\left({ }_{40} C_{2}=(40 \times 39) / 2=780\right)$. These intervals yield more than three hundred thousand pairs of intervals for comparison $\left({ }_{780} \mathrm{C}_{2}=(780 \times 779) / 2=\right.$ 303,810). Highlighting particular kinds of intervals (i.e., unisons, octaves, etc.) facilitates greatly a potentially difficult search for pattern among the forty tones. Valid distinctions between kinds of intervals and types of instruments also serve to simplify considerably the technical account that follows. Further compression arises from treating groups of tone-pairs statistically.

By means of statistics, one can secure several overviews of an ensemble, both as a whole and in its various parts. Such overviews can summarize thousands of comparisons of particular tone-pairs and also allow one to determine the degrees to which certain similarities and differences in interval size are significant. The following account uses statistics as a means of simplifying the discussion and assessing the significance of certain distinctions.

Another potential difficulty arises from the largely non-numerical approach to tuning that seems always to have been characteristic of Indonesian music theory. In Western, Middle Eastern, and East Asian cultures, there have been centuries-long traditions of specifying numerically the ideal sizes of pitch-intervals in various scales and modes. For example, the ratios $2 / 1$ and $3 / 2$ have been widely, and rather persistently, employed in these cultures to characterize the proportions of string-lengths that produce perfect octaves and fifths, respectively. By contrast, there have been no parallel approaches to tuning in Indonesian culture until relatively recent decades.

To be sure, Machjar Koesoemadinata (New Indonesian: Magyar Kusumadinata) has advanced numerically formulated proposals for future tunings in West Java. ${ }^{8}$ However, these proposals do not tally closely with the tunings of actual ensembles that have continued to be used and have not been adopted widely as tuning standards. ${ }^{9}$ Additionally, a government gamelan factory in Surukarta on the island of Java has used sets of tuned key plates for the quick production of new instruments. ${ }^{10}$ However, the measured frequencies of these slabs do not appear to embody a particularly privileged pattern of specific frequency ratios and do not accord especially closely with the frequencies of other ensembles. Instead of being viewed as fully definitive or precisely authoritative, Kusumadinata's proposals and the factory's key plates can be regarded as comprising sufficient, but not necessary, conditions for arriving at traditional tunings.

Throughout the world, tuning theories framed in terms of specific frequency ratios have comprised sufficient, but not necessary, conditions for producing the tones of particular scales and modes. For instance, studies of the actual frequencies produced on pianos tuned by Western experts reveal wide diver-

8Raden Machjar Angga Koesoemadinata, Ilmu seni-raras: ilmu musik Indonesia alsi (Jakarta: Pradnja Paramita, 1969), 27-46.

9 Max Harrell, "West Java, Classical Music," in The New Grove Dictionary of Music and Musicians, ed. Stanley Sadie (London: Macmillan, 1980), 9:207-10, esp. 210; cf. Andrew N. Weintraub, "Theory in Institutional Pedagogy and 'Theory in Practice' for Sundanese Gamelan Music," Ethnomusicology 37, no. 1 (1993): 29-39.

10Donald Lentz, The Gamelan Music of Java and Bali: An Artistic Anomaly Complementary to Primary Tonal Theoretical Systems (Lincoln, Nebraska: University of Nebraska Press, 1965), 54. 
gences from the various prescriptions of European tuning theories. ${ }^{11}$ Careful studies in music psychology indicate that Westerners categorize intervals as being, for example, major thirds and minor thirds within spans of tolerance so large as to prevent one from distinguishing whether one particular tuning prescription (e.g., just intonation) or another (e.g., equal temperament or Pythagorean tuning) is operative. ${ }^{12}$ Even quite highly trained Western musicians are rather unreliable in distinguishing between, for instance, wide minor thirds and narrow major thirds. ${ }^{13}$ Parallel findings have emerged in studies of tuning and perception outside the West. ${ }^{14}$

If instruments are tuned as closely as possible to the frequency ratios specified by various orthodox tuning theories that have arisen within particular cultures, they generally "pass" as being "in-tune" relative to their respective musical traditions. However, many more particular tunings than have been formulated by theorists-in principle, an infinite number-would serve quite well in any culture as means of instantiating particular scales or modes. In other words, a particular numerical formulation is just one among many possible ways of realizing a particular tonal system, and hence, a sufficient, but not necessary, condition for producing the desired intervals.

The following account makes much use of specific numbers (i.e., of cents) to describe the frequency ratios of intervals. In general, numbers of this sort should be regarded merely as tools for describing such minimally quantitative relations between intervals as "is larger than" and "is smaller than." Arguably, people hear one pair of tones as being intervallically similar to, different from, larger than, or smaller than, another-or even larger or smaller in varying degrees (e.g., as conveyed by such modifiers as "much" and "slightly"). The interpretation of frequency measurements offered below runs along such nonnumerical, not fully quantitative, perceptual lines, even though it is reached through frequency measurements cast in quite precise, specific numbers. In this regard, the virtually non-numerical tradition of tuning theory in Indonesia can be appreciated as opportune rather than problematic, because it forces scholars

11 E.g., R.W. Young, "Inharmonicity of Plain Wire Piano Strings," Journal of the Acoustical Society of America 24 (1952): 267-73, and D.W. Martin and W.D. Ward, "Subjective Evaluation of Musical Scale Temperament in Pianos," Journal of the Acoustical Society of America 33 (1961): 582-85.

12E.g., Donald E. Hall and Joan Taylor Hess, "Perception of Musical Interval Tuning," Music Perception 2, no. 2 (1984): 166-95.

13E.g., Jane A. Siegel and William Siegel, "Categorical Perception of Musical Intervals: Musicians Can't Tell Sharp from Flat," Perception and Psychophysics 21, no. 5 (1977): 399-407, and E.M. Burns and W.D. Ward, "Categorical Perception-Phenomenon or Epiphenomenon: Evidence from Experiments in the Perception of Melodic Intervals," Journal of the Acoustical Society of America 63 (1978): 456-68.

14E.g., Nazir A. Jairazbhoy and A.W. Stone, "Intonation in Present-Day North Indian Classical Music," Bulletin of the School of Oriental and African Studies 26, no. 1 (1963): 119-32; Johanna Spector, "Classical 'Ud Music in Egypt with Special Reference to Maqamat," Ethnomusicology 14, no. 2 (1970): 243-57; E.M. Burns, "Octave Adjustment by Non-Westem Musicians," Journal of the Acoustical Society of America 56 (1974): S5 [Abstract]; Peter R. Cooke, "Exploring Musical Pitch Systems," International Council for Traditional Music, U.K. Chapter Bulletin 27 (1990): 6-24, and "Report on Pitch Perception Experiment Carried Out in Buganda and Busoga (Uganda) August 1990," Ethnomusicology Research Digest [electronic periodical] 30 (1990); and Bernard Bel, "Equiheptatonic Tuning," Ethnomusicology Research Digest 38 (1991). 
to re-think critically the very bases of interval perception, which here are regarded as being non-numerical.

Since Ellis's pathbreaking work more than a hundred years ago, ethnomusicologists have generally characterized the main scale dealt with here, namely, slendro, as "equipentatonic." Ethnomusicologists have regarded each of slendro's five scale degrees as being separated-at least ideally or in theory-from its neighbouring, adjacent scale degrees by a fifth of an octave (ideally, $1200 / 5=240$ cents). Nevertheless, Ellis's original study indicated that such an ideal or theoretical value corresponded only approximately to actual practice. Subsequent studies have shown that there can be considerable deviation from the fifth-octave, 240-cent ideal within and among instruments on which slendro pieces traditionally have been played. All the same, it is clear that slendro tunings are not capricious. For example, slendro instruments that are recognized, within Indonesian musical culture, as being "in-tune" never seem to feature any intervals that are quite as small as some of the intervals that regularly appear in the other principal scale of Indonesia, namely, pelog.

Both Indonesian scholars (e.g., Kusumadinata) and such Western ethnomusicologists with many years experience in Indonesian musical culture as Jaap Kunst ${ }^{15}$ have regularly employed European staff-notation to write down both slendro and pelog pieces. Such scholars have never specified, for slendro, intervals smaller than a whole tone, which ideally, in Western equal temperament, comprises 200 cents. By contrast, in their notations of pelog pieces, they have consistently employed intervals of a semitone, which correspondingly comprise (ideally, again) 100 cents.

A usual "translation" of pelog into Western staff-notation employs the notes E, F, G, B, and C in all octaves, with occasional use of the notes A and D to indicate "extra," "chromatic" tones. Corresponding translations of slendro generally employ such anhemitonic pentatonic sets as G, A, B, D, and E, and G, A, $\mathrm{C}, \mathrm{D}$, and $\mathrm{E}$. Other renderings, discussed below, have appeared as well, and generally these have not used Western semitones to convey the sounds of slendro. Once again, the problem to be confronted involves discerning and accounting for unity within relatively great amounts of diversity. In particular, one seeks to know what sorts of boundaries there might be on the generally large "seconds" in slendro that distinguish them from their small pelog counterparts and serve to distinguish slendro as a scale.

The final major problem to be considered here involves Western responses to Indonesian tunings. In other contexts, one might seek to determine musical norms entirely within a single musical tradition. A comprehensive study of such an ensemble as is highlighted here requires that one also take into account possible ways in which a musical system might be received outside the culture where it originated. In the area of avant-garde composition and improvisation, Indonesian creators have worked literally side-by-side with Western counterparts in making music for, and on, traditional Indonesian instruments. More-

15Jaap Kunst, Music in Java, 3rd enl. ed., E.L. Heins, ed. (The Hague: Martinus Nijhoff, 1973; 1st ed., 1934; 2nd ed., 1949). 
over, such ensembles as gamelan angklung have become mainstays in Western university curricula. Indeed, the particular orchestra analyzed intensively below was brought from Bali specifically for use by North American university students.

Ways in which transplanted Indonesian ensembles might be "heard" by Western audiences and performers are indicated, in part, by the "translation" into something like equal temperament that seems to be implied in notating music for Indonesian instruments according to Western conventions. However, such notations are only partial, and, if used at all in practice, often serve only as a guide for the leader or other musicians in an ensemble, or as a reference text between rehearsals or performances. Unnotated in such scores is a large amount of gradated tuning detail of the sorts outlined above. Within such detail lies a potentially important opportunity for advanced, "bi-musical" ear training on the part of Western musicians and listeners who constitute, in a certain sense, a "host" culture for various transplanted musical systems of Indonesia.

Western listeners and performers might respond to a slendro ensemble as though it were merely "out of tune" relative to equal temperament. However, a later section of this study shows how Westerners might learn to respond in ways concordant with the musical systems that appear to underlie traditional Indonesian tunings. Such responses appear to have their basis in perceptual processes that are shared across cultures. For example, the patterns of "mis-tuned" unisons and octaves one finds in Indonesian instruments are quite regular and are also consistent with findings in psychoacoustics that appear to be valid cross-culturally. As well, the great variety of particular tunings that are recognized within Indonesian musical culture as valid instances of slendro and pelog can be subsumed by current formulations in music theory which are universal in scope. In these ways, both Indonesian and Western tunings can be regarded as special cases of structures and processes that are found throughout the world.

The following sections clarify points raised in the preceding survey of problems. In conformity with the hierarchy of difference and similarity outlined above, separate sections deal with individual tones, unisons, octaves, other intervals, and slendro as a scale. The discussion of these topics is cumulative and leads to a proposal for gamelan-related musicianship instruction and a review of the status of numbers in theories of music. The centrepiece of the account consists of frequency measurements taken on a Balinese gamelan angklung brought to North America for use by university students. As a background to the discussion of the frequency measurements, separate sections deal with the instruments in this ensemble and the method employed in measuring its frequencies. To the best of my knowledge, detailed tone measurements of Balinese fixed-frequency instruments have only been published before by Kunst, Hood, and Ruby Ornstein, ${ }^{16}$ even though other aspects of Balinese

16 Jaap Kunst, De Toonkunst van Bali: Beschouwingen over Oorsprung en Beinvloeding, Composities, Notenschrift en Instrumenten (Welvereden: G. Kolff \& Co., 1925); Hood, "Sléndro and Pélog Redefined"; and Ruby Ornstein, Gamelan Gong Kebjar: The Development of a Balinese Musical Tradition (unpub. Ph.D. diss., University of California at Los Angeles (Music), Ann Arbor: University Microfilms, 72-2950, 1971), 2 vols. 
gamelan have been studied intensively for several decades. Since the central issue of this study, namely, distinguishing most effectively between similarities and differences in gamelan tunings, has persisted for more than a century in ethnomusicological studies, these are discussed at the conclusion.

\section{The Universe Gamelan Angklung}

The present report focuses on a gamelan angklung orchestra that has been used in the University of Pittsburgh's "Semester at Sea" programme for more than a decade. Since 1982, undergraduate students in the SAS programme have taken instruction in gamelan angklung performance, along with three or four other half-courses in such areas as world music, Southeast Asian music, comparative literature, cultural anthropology, religious studies, international business, global economics, and marine biology. All of these courses, and many others of a worldwide nature, have been held regularly, both aboard the S.S. Universe as it has sailed around the world, twice each year, and onshore, by way of faculty-led practica held in various countries where the ship has docked.

The SAS programme's gamelan angklung is housed permanently on board the Universe. Its faculty leaders have included, since 1982, myself, Joseph Pacholczyk, Patricia Matusky, Robert Kauffman, Peggy Caton, Gertrude Robinson, Usopay Cadar, JaFran Jones, Peter Manuel, Roderic Knight, Max Brandt, Ted Solis, I Wayan Ledra, Ahmet Yurur, and the late James Koetting. The orchestra was purchased for SAS through Philip Sonnichsen from I Wayan Suweca in 1982.

Suweca described the orchestra in 1982 as having been made by Pande at Klungkung in Bali. Suweca went on to relate that it had a "very sweet sound" and "old key[s]," and that it would be a good idea to house the orchestra permanently on the ship "because the tun[ing will] never change again" (i.e., because the metal had settled and the tuning, thus, had stabilized). Suweca also indicated in 1982 that the ensemble's instruments were "paint[ed] with gold paint and carve[d]" (i.e., in the traditional way). In sum, not only in tuning but also in appearance, the Universe ensemble, as described by Suweca, is fully traditional. ${ }^{17}$

Several of those who have led instruction on the Universe gamelan's instruments have remarked without prompting on its delightful sound. Such directors of the orchestra as Roderic Knight and Patricia Matusky have assessed the Universe gamelan as "a beautiful little set of instruments" and "a great little ensemble." 18 Nothing in Suweca's original account of 1982 conflicts with my own experience of the instruments during the January-May semester of 1992 when I led a course in angklung performance on board the Universe and undertook the tone measurements reported below.

17Max Brandt, personal communication, August, 1992, based on correspondence with Philip Sonnichsen and I Wayan Suweca, September, 1982.

18Max Brandt, personal communication, August, 1991, based on correspondence with Roderic Knight, [n.d.: December, 1985?], and Patricia Matusky, May, 1990. 
The core of the Universe ensemble consists of ten keyed bronze metallophones: eight gangsa instruments that comprise four kantils and, an octave lower, four genders, plus two jegogans, tuned yet another octave lower. Each of these instruments produces four tones of the five traditionally used in slendro. In the Universe gamelan, these four tones correspond quite closely to $G, A, B$, and $D$ in Western notation. The genders' $G$ sounds quite close to the $G$ on the second line of the treble clef (cf. Figure 1, and Examples 1 and 2). ${ }^{19}$

\section{Frequency Measurements}

The fundamental frequencies of individual tones in the Universe gamelan were measured by means of a Stroboconn $61 \mathrm{~A}$ tuner. Tones sent into the Stroboconn produce clockwise and counterclockwise rotations of striated concentric circles. The darkness of the striation and the slowness of the rotation vary directly with the amount of energy at a particular frequency. Twelve sets of concentric circles correspond to pitch classes C, C-sharp, ... B. The eight concentric circles for each set of pitch-classes comprise eight octaves of the pitch class, low to high corresponding to the progression from inner to outer circles. A separate dial allows one to vary the absolute frequency of the entire set of twelve pitch classes through 50 cents above and below the zero mark, which is set, by default, at A440.

19 Also available were a pair of two-headed drums (kendang); a vertical hanging gong (kempur) sounding close to A-flat, top line of the bass clef ( $200.7 \mathrm{cps}, 241$ cents-cf. Figure 3a, below); a smaller vertical hanging gong (kempli) that produces a very sharp version of the jegogans' D (302.6 cps, 852 cents); another, horizontally suspended gong (kelenang) supported by a wooden stand and sounding somewhat higher than an octave above the kantils' $G$ ( $1789 \mathrm{cps}, 3929$ cents); and a single set of five bronze cymbals (rincik) tuned to the four tones of the keyed instruments, fixed horizontally in overlapping fashion to the back of a turtle-shaped stand and struck with a similar pair of bronze hand cymbals. Not employed, because it was incompete and in need of repair, was a pair of small double-gongs (reyong) tuned closely to the G, A, B, and D of the kantils, respectively, and mounted on the ends of dumb-bell-shaped wooden holders. The latter instruments are not invariably used in pieces for angklung and are not reported on further here.

Descriptions of the instruments and their traditional playing techniques appear in Colin McPhee, Music in Bali: A Study in Form and Instrumental Organization in Balinese Orchestral Music (New Haven: Yale University Press, 1966) and in the respective articles on individual instruments in Stanley Sadie, ed., The New Grove Dictionary of Musical Instruments, 3 vols. (London: Macmillan, 1984). Wayne Vitale, "Gamelan Angklung in Denver," Balungan 4, no. 2 (1990): 32-33 provides an account of angklung in a contemporary avant-garde Western setting. Mantle Hood and Jose Maceda outline the cultural context of traditional angklung performance in Music (Leiden: E.J. Brill, 1972), 25, as do McPhee, "Angkloeng Gamelans in Bali," Djawa 17 (1937): 322-50, and Ernst Schlager, Rituelle Siebenton-Musik auf Bali, Part One, ed. Hans Oesch (Bern: Francke Verlag, 1976), 45. Recordings and photos of various kinds of angklung ensemble appear on the following, commercially available releases: David Lewiston, comp., The Balinese Gamelan: Music from the Morning of the World (Nonesuch Explorer Series, mono H-2015, stereo H-72015), side 2, band 2, and photos 8 and 9; Doreen Powers, comp., Scintillating Sounds of Bali (Lyrichord Stereo LLST 7305), side 1, band 2, and photos 1, 3, and 4; and Louis Berthe, comp., Barong: drame musical balinais/A Balinese Musical Drama (Disques Vogue, VG 403, LD 763), side 2, band 3. The angklung ensemble discussed here is not to be confused with the archaic, 5-tone angklung orchestra that employs tuned bamboo rattles and that has been found mostly in the north of the island. McPhee, Music in Bali, and Ruby Ornstein, "The Five-Tone Gamelan Angklung of North Bali," Ethnomusicology 15, no. 1 (1971): 71-80 provide descriptions of this, apparently original, form of the angklung ensemble. Commercial recordings of it appear on Ruby Omstein, comp., Gamelan Music of Bali (Lyrichord [mono] LL179, stereo LLST 7179), side 2, bands 1 and 2. 
pengumbang (lower of beating pairs)

pengisep (upper of beating pairs)

jegogans (large metallophones):

jegogan 1

jegogan 2

gangsas (small metallophones):

gender 1

gender 3

kantil 1

kantil 3

pengumbang gangsas play

lower, pengumbang versions of:

all tones (G,A, B, and D)

in normal performance

and

lower, nyangsih tones ( $G, A)$

in broken kotekan gender 2

gender 4

kantil 2

kantil 4

pengisep gangsas play

upper, pengisep versions of:

all tones (G, $A, B$, and $D$ )

in normal performance

and

upper, molos tones (B, D)

in broken kotekan

Figure 1: Seating arrangement of metallophones in gamelan angklung (after McPhee, 1966; used with permission of Yale University Press). Rubrics indicate pengumbang ( 1 and 3 ) and pengisep ( 2 and 4) instruments paired to produce penjoreg-beating, as well as divisions between instruments in kotekan style: lower, nyangsih (1 and 3) playing $G$ and $A$ in Example 2 (above); upper, molos ( 2 and, in broken style, 4) playing B and D. In woven-style kotekan, intermediary, kilitan instruments (3) weave together low, pengumbang versions of $G, A, B$ and $D$ in lines combining tones from lower, nyangsih and upper, molos parts.

Once one has "caught" a relatively slowly rotating, darkly striated circle in one of the twelve sets of concentric circles, one can "hedge in" the final reading by gradually reducing the span between positions of the cents-dial that produce clockwise and counterclockwise rotations. A clockwise reading indicates that the stronger, more energetic portion of the tone (or one of its partials) corresponds to a higher frequency than the current setting of the cents-dial; a counterclockwise reading indicates that more than half the energy in this frequency region is lower (i.e., in frequency) than the current setting. On the way to a final reading of, for example, A3-29 cents (where $A 3=220 \mathrm{cps}$ ), one might have passed through A3, A3-50, A3-10, A3-40, A3-20, A3-35, A3-25, yielding alternating clockwise and counterclockwise rotations, respectively, with a gradual darkening and slowing down of the rotating striations in the display. Further fine-tuning results in increasing proximity to a final setting that would produce, ideally, a very dark and completely static striated circle in the A3 region of the A pitch class. Remaining clockwise and counterclockwise motions were reduced to the level of a single cent and cps by adjusting the setting until the rotations comprised less than a single striation over a two-second period. 
Readings were taken on one day and checked the next. It must be stressed that from the first stages in a study of this sort, one is concerned with spans of tolerance and probabilities. In this way, a statistical approach to the measurements is methodologically appropriate. Much of statistical theory, including the parameters employed in this study, was developed in order to assess the results of measurements in general, giving due allowance to the possibility of random errors.

\section{Spectrum and Tuning}

As indicated above, Vetter has quoted Schneider to the effect that spectral analysis of gamelan tones reveals chaotic structures, and has called into question the sorts of precise measurements reported here and in the mainstream of gamelan-tuning studies for more than a century. In Vetter's words, "there is no clearly discernible fundamental but a rather dense and inharmonious spectrum that gives rise to auditory uncertainty."20 Having employed the instruments of the Universe gamelan in instructing undergraduates for more than three months, I can assert that there is not much auditory uncertainty beyond what is covered by the truism that all physical and perceptual processes are ultimately probabilistic. Non-music majors displayed little difficulty in matching the instruments' tones with their voices throughout a curriculum that mixed instrumental performance and singing.

Sound Tools software on a Macintosh computer was used to assess the acoustical portion of Vetter's assertion, which forms a basis for his highly skeptical study. The software produced several graphic displays of each tone on the keyed metallophones. Far from the results being chaotic or perceptually uninterpretible, there was remarkable uniformity and coherence among the various tones.

Common to all tones was a continuous, relatively narrow energy peak at the frequency that had been been measured as the fundamental by the Stroboconn. The beginning of this energy peak appeared at some point during the first approximately 50 milliseconds, that is, around $1 / 20$ th second, that constituted much of the tone's attack. This fundamental lasted as long as the key was vibrating and the tone could be heard (in some instances for longer than a minute on these remarkable instruments). Throughout this period, the fundamental remained extremely constant in frequency and was also the spectral component of greatest intensity. Within this uniform framework, there were consistent, distinct patterns (Figure 2).

In jegogan tones, the fundamental appeared at the very outset in conjunction with energy at immediately surrounding, especially lower frequencies. These surrounding frequencies and the beginning portion of the fundamental effectively constituted the tone's attack, after which any other frequencies almost entirely disappeared. In gender tones, the fundamental appeared shortly after a slight burst of energy in a band located at substantially lower frequencies, which constituted the beginning of the attack. As well, in the gender tones, there was,

20Vetter, “A Retrospect on a Century of Gamelan Tone Measurements," 217-27. 

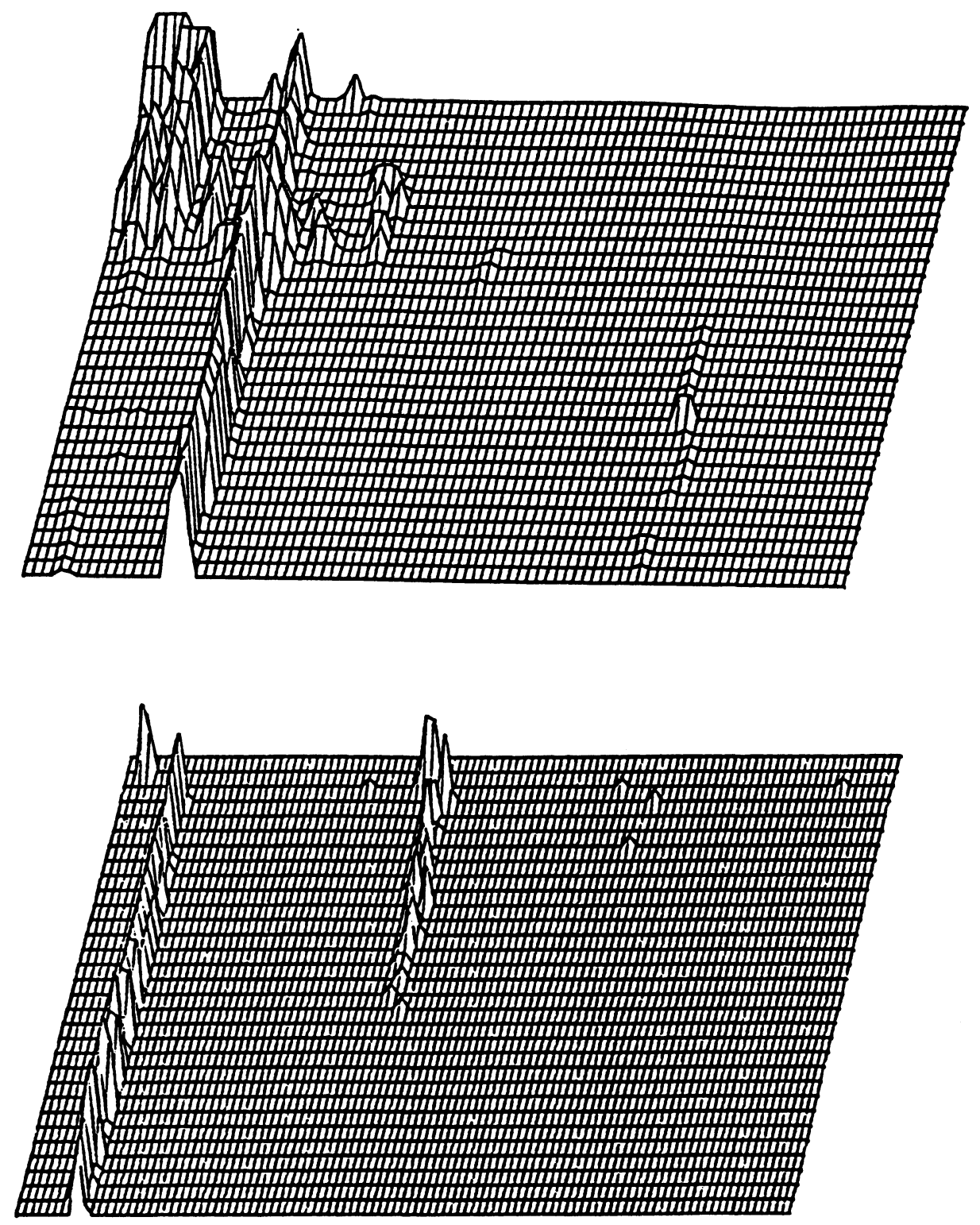

Figure 2 (cont'd): Graphic displays of individual tones in Universe gamelan. Left-to-right on grid corresponds to increasing frequency; top-to-bottom, time, from earlier to later; amount of height above plane of frequency and time, amplitude. Top: G3 of jegogan 1 (300 milliseconds, from 90 to $1015 \mathrm{cps}$, low portion of attack in upper left corner, cross-section of fundamental at $300 \mathrm{msec}$ in foreground), bottom: G4 of gender 1 (400 msec, 0 to 17,200 cps), and on next page, top: G5 of kantil 1 (300 msec, 0 to $18,920 \mathrm{cps})$, bottom: extremely high-resolution graph of tone AS of kantil 2 (250 msec, 750 to $1050 \mathrm{cps}$ ). 

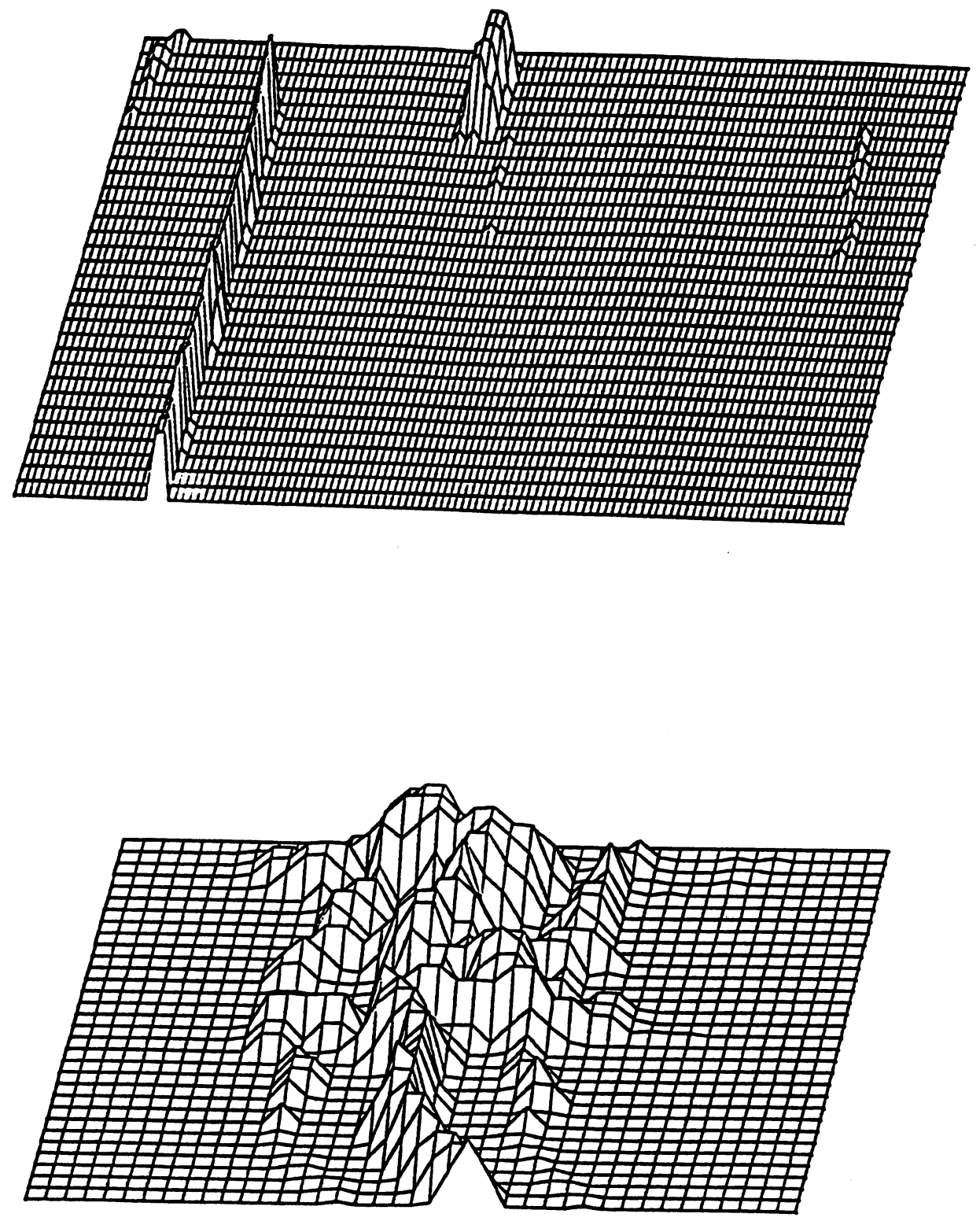

Figure 2 (concl'd): 
just before, and/or during, the first several milliseconds of the fundamental, a burst of energy in one or two regions much higher than the fundamental. Kantil tones displayed much the same spectral pattern as gender tones, with a distinct additional tendency for the upper frequencies to overlap temporally the low initial frequencies and the subsequent fundamental. Common to all three sorts of tones, but uniformly found in none of the three groups, were individual, extremely brief, low-energy bursts above or, more rarely, below, the fundamental. These small bursts were consistently quite separate in time and frequency from the much more energetic bursts that accompanied the attacks.

The distinct patterns found during the attack and immediately thereafter in the various kinds of instruments correspond to timbral distinctions among the tones. The initial portion of a tone long has been known to constitute the most important segment for identifying, and distinguishing between, various kinds of instruments. For example, E.J. Saldanha and J.F. Corso report that listeners identify musical instruments correctly more often if presented with the beginning portion than if presented with the middle and/or end. ${ }^{21}$

That the fundamentals of all three kinds of tone involve at least a narrow spread in frequency (conveyed in Figure 2 by the width of the ridge for each fundamental) is a manifestation of Heisenberg's Uncertainty Principle, according to which, no wave (e.g., a sine-tone) can converge on a single value (which would correspond to a vertical line segment rather than the triangular or trapezoidal cross-section for the fundamentals in Figure 2 until an infinite amount of time has passed. ${ }^{22}$ Instead, all periodic waves, even sine-tones, comprise at their outsets quite wide bands of energy which might narrow considerably, but always necessarily only finitely, over time. ${ }^{23}$

Extreme, "close-up" views of tones produced by all three kinds of instruments reveal jagged, "mountain-range" patterns. Plausibly, graphic displays of such seemingly chaotic energy bursts were what Schneider observed and Vetter reported as a basis for his skeptical outlook on tone measurements. However, it has long been known that attacks generally feature such seemingly erratic bursts as a prelude to the much more stable portions that follow. ${ }^{24}$ Further, because of the Uncertainty Principle, relatively wide side-bands arise at the beginning of any wave whatever. Most important, such patterns are displayed only if the resolution of time and frequency is quite high, whereas the work of Nobel laureate Georg von Békésy (e.g., as surveyed in his account of Sensory Inhibition $)^{25}$ has emphasized that the ear responds to frequencies by means of an

21E.L. Saldanha and J.F. Corso, "Timbre Cues and the Identification of Musical Instruments," Journal of the Acoustical Society of America 36 (1964): 2021-26.

22Cf. Stanley Smith Stevens and Hallowell Davis, Hearing: Its Psychology and Physiology (New York: American Institute of Physics for the Acoustical Society of America, 1938; repr. 1983), 103-4, and G.W. Stewart, "Problems Suggested by an Uncertainty Principle in Acoustics," Journal of the Acoustical Society of America 3 (1931): 325-30.

23Cf. Robert Erickson, Sound Structure in Music (Berkeley: University of California Press, 1975), 61-62.

24E.g., Erickson, Sound Structure in Music, 61-68.

25 Georg von Békésy, Sensory Inhibition (Princeton, N.J.: Princeton University Press, 1967), 175-214. 
averaging, focusing process-rather like the convergent method of taking Stroboconn measurements.

There was no particular pattern to the generally brief, ancillary energy bursts that were found in some of the tones. Had these been relatively persistent, intense, and located at frequencies close to, but not coincident with, particular multiples of their fundamentals, they might have influenced considerably the pitch-perception of individual tones in such a way as to call into serious question an identification of the pitches of the tones with their focal, fundamental frequencies. ${ }^{26}$ Even taking into account their attack portions and relatively short, weak, later bursts, all the spectra examined in this study lead one to identify the perceived pitches of these tones with their fundamental frequencies. The tones examined resemble quite closely those produced by tuning forks, dominated as they are by an energetic, sine-tone-like segment at the fundamental frequency. Characteristics of the instruments' attacks appear to be related to whether a wooden mallet is used, producing a clear "ping" at the outset of a gender or kantil tone, or a cloth beater, which yields a dull "thung" in jegogan tones.

The Uncertainty Principle accounts for (a) the persistence of considerable width in the cross-sections of the fundamentals (clearly evident in the bottom left portions of the graphs in Figure 2), and, correspondingly, (b) the remaining rotations of the circles that persisted at the conclusion of the Stroboconn measurements-both of these within relatively small spans of frequency. One can understand the Stroboconn as capturing, or hedging in, at the level of a single cent and cps, a value corresponding to the average frequency at which energy occurred in the fundamental, to which the ear, in turn, responds most energetically. These observations justify the view of unison- and octave-interaction outlined below and validate the mainstream of Indonesian tuning studies, as contrasted with Vetter's skeptical outlook. Figure 3 summarizes the results of the tuning measurements taken on the Universe gamelan. These results are reported in terms of both fundamental frequencies (expressed in cps) and ratios between such frequencies (expressed in cents).

\section{Unisons and Beats}

Tones that are quite close in frequency to an absolute unison produce audible beats of various sorts. In traditional performance, the two jegogans play in unison throughout a piece. Similarly, except for interlocking, kotekan passages, all four genders play in unison as do all four kantils, an octave higher (cf. Ex. 1, above). In each instance, the respective tones on the instruments can be identified readily by the fact that each key corresponds to a unique numeral $(1,2,3$, or 4$)$ in the traditional cipher notation used in Bali and a distinctive syllable in the traditional solmization system.

One jegogan (2, in Fig. 3 ) is typically tuned somewhat higher (pengisep) than the other (1: pengumbang), and the same holds for pairs of genders ( $2 \mathrm{vs.} 1$, and $4 v s .3$ in Fig.3), and for pairs of kantils (again, $2 v s .1$, and $4 v s .3$ ). In this way,

26Cf., for example, E. Terhardt and H. Fastl, "Zum Einfluss von Stortonen und Storgerauschen auf die Tonhöhe von Sinnestonen," Acustica 25 (1971): 53-61. 


\begin{tabular}{|c|c|c|c|c|}
\hline Instruments & $\begin{array}{c}\text { Cipher } \\
\text { and key no. }\end{array}$ & $\begin{array}{c}\text { Westem } \\
\text { letter-name }\end{array}$ & Cents $(F \# 3=0)$ & Cps $(\mathrm{Hz})$ \\
\hline \multirow{4}{*}{ jegogan 1} & 1 & G3 & 72 & 192.9 \\
\hline & 2 & A3 & 271 & 216.3 \\
\hline & 3 & B3 & 463 & 241.7 \\
\hline & 4 & D4 & 766 & 288.0 \\
\hline \multirow[t]{4}{*}{ jegogan 2} & 1 & G3 & 124 & 198.7 \\
\hline & 2 & A3 & 333 & 224.2 \\
\hline & 3 & B3 & 517 & 249.4 \\
\hline & 4 & D4 & 809 & 295.2 \\
\hline \multirow[t]{4}{*}{ gender 1} & 1 & G4 & 1344 & 402.1 \\
\hline & 2 & A4 & 1535 & 449.0 \\
\hline & 3 & B4 & 1731 & 502.8 \\
\hline & 4 & D5 & 2039 & 600.7 \\
\hline \multirow[t]{4}{*}{ gender 2} & 1 & G4 & 1380 & 410.5 \\
\hline & 2 & A4 & 1558 & 455.0 \\
\hline & 3 & B4 & 1753 & 509.2 \\
\hline & 4 & D5 & 2060 & 608.0 \\
\hline \multirow[t]{4}{*}{ gender 3} & 1 & G4 & 1344 & 402.1 \\
\hline & 2 & A4 & 1538 & 449.8 \\
\hline & 3 & B4 & 1733 & 503.4 \\
\hline & 4 & D5 & 2039 & 600.7 \\
\hline \multirow[t]{4}{*}{ gender 4} & 1 & G4 & 1381 & 410.8 \\
\hline & 2 & A4 & 1566 & 457.1 \\
\hline & 3 & B4 & 1753 & 509.2 \\
\hline & 4 & D5 & 2061 & 608.4 \\
\hline \multirow[t]{4}{*}{ kantil 1} & 1 & G5 & 2536 & 800.5 \\
\hline & 2 & A5 & 2790 & 927.0 \\
\hline & 3 & B5 & 2980 & 1034.5 \\
\hline & 4 & D6 & 3293 & 1239.5 \\
\hline \multirow[t]{4}{*}{ kantil 2} & 1 & G5 & 2550 & 807.0 \\
\hline & 2 & A5 & 2793 & 928.6 \\
\hline & 3 & B5 & 2993 & 1042.3 \\
\hline & 4 & D6 & 3299 & 1243.8 \\
\hline \multirow[t]{4}{*}{ kantil 3} & 1 & G5 & 2538 & 801.4 \\
\hline & 2 & A5 & 2782 & 922.7 \\
\hline & 3 & B5 & 2982 & 1035.7 \\
\hline & 4 & D6 & 3297 & 1242.4 \\
\hline \multirow[t]{4}{*}{ kantil 4} & 1 & G5 & 2555 & 809.3 \\
\hline & 2 & A5 & 2801 & 932.9 \\
\hline & 3 & B5 & 2998 & 1045.3 \\
\hline & 4 & D6 & 3303 & 1246.7 \\
\hline
\end{tabular}

Figure 3a: Fundamental frequencies of keyed metallophones in Universe gamelan. 


\begin{tabular}{|c|c|c|c|c|c|c|c|c|c|c|c|c|}
\hline \multirow[t]{2}{*}{ Jegogans } & \multicolumn{7}{|c|}{ Genders } & \multicolumn{2}{|c|}{ Kantils } & \multirow[b]{2}{*}{ A5 } & \multirow[b]{2}{*}{ B5 } & \multirow[b]{2}{*}{ D6 } \\
\hline & G3 & A3 & B3 & D4 & G4 & A4 & B4 & D5 & G5 & & & \\
\hline \multicolumn{13}{|l|}{ normal: } \\
\hline & 195.8 & 220.3 & 245.6 & 291.6 & 406.4 & 452.7 & 506.2 & 604.5 & 804.5 & 927.8 & 1039.5 & 1243.1 \\
\hline & 98 & 302 & 491 & 788 & 1362 & 1549 & 1743 & 2050 & 2545 & 2792 & 2988 & 3298 \\
\hline \multicolumn{13}{|l|}{ kotekan: } \\
\hline \multirow[t]{2}{*}{ broken: } & & & & & 402.1 & 449.4 & 509.2 & 608.2 & 801.0 & 924.9 & 1043.8 & 1245.3 \\
\hline & & & & & 1344 & 1537 & 1753 & 2060 & 2537 & 2786 & 2996 & 3301 \\
\hline \multirow[t]{2}{*}{ woven: } & & & & & & & 507.3 & 605.7 & & & 1041.1 & 1244.3 \\
\hline & & & & & & & 1746 & 2053 & & & 2991 & 3300 \\
\hline
\end{tabular}

Figure 3b: Best estimates of "the pitches" of the Universe gamelan according to three possibilities of performance: normal and kotekan, the latter in broken and woven forms. Upper rows of all pairs of entries are in cps; lower rows, in cents (F-sharp3 = 0 cents). 
traditional Balinese tuning aims at producing beating (penjoreg) between pairs of instruments. In the Universe gamelan, the jegogan unisons tend to be about the same in size as the unisons between genders 1 and 2 , and 3 and 4, that is, about $7 \mathrm{cps}$. Similarly, kantils 1 and 2 , and 3 and 4 tend to produce unisons that are fairly close to 7 cents. The overall range of such penjoreg unisons is from 4.3 to $10.2 \mathrm{cps}$, with a single exception comprising $1.6 \mathrm{cps}$. By contrast, genders 1 and 3 , and 2 and 4 produce much smaller unisons, from zero to $2.1 \mathrm{cps}$, all but one being of $0.8 \mathrm{cps}$ or less. The corresponding pairs of kantils (i.e., 1 and 3 , and 2 and 4 , again) tend also to produce relatively small unisons, namely, from 0.9 to $4.3 \mathrm{cps}$, most being $2.3 \mathrm{cps}$ or larger.

Statistically, penjoreg-unisons on all three groups of instruments are significantly similar to (i.e., not significantly different from) each other and significantly larger than the corresponding groups of non-penjoreg-unisons, and significantly larger than zero cps. Groups of non-penjoreg-unisons on the kantils are significantly similar to each other and significantly larger than the corresponding groups of non-penjoreg unisons on the genders. The non-penjoreg gender unisons are significantly similar to each other and, like the non-penjoreg kantil unisons, significantly larger than zero cps. ${ }^{27}$ In principle, a sufficiently acute listener might make further distinctions within these broad groupings of statistical similarities and differences, and a relatively insensitive listener, fewer. Nonetheless, a statistical treatment has the merit of providing tests of distinctions that have been made in other tuning studies and in music psychology.

The average penjoreg (about $7 \mathrm{cps)}$ throughout the Universe ensemble corresponds to the estimate of "roughly 7 or $8 \mathrm{cps"} \mathrm{that} \mathrm{Ornstein} \mathrm{reports} \mathrm{for}$ gamelan gong kebyar in Pliatan, Bali. The rates she records extend similarly from an exceptionally low 1.9, through 4.5 and 5.0 to her fastest value, $10.5 \mathrm{cps}$, a range of 6.3 to 9.3 constituting the bulk of her measurements. ${ }^{28} \mathrm{Hood}$ reports similar differences of six to seven cps for corresponding keys of all the paired instruments in his own gender wayang quartet "Pemungkah" and in UCLA's large gamelan gong kebyar "Sekar Anjar." As well, Hood provides precise figures that range from 6.5 to $7.5 \mathrm{cps}$ for five pairs of tones on the two jegogans of the latter ensemble. ${ }^{29}$ All these measurements bear out and amplify the statement of Balinese musician Tjokorda Agung Mas that the number of beats between the tones of paired instruments is constant in individual ensembles. ${ }^{30}$

27Employed in the statistical tests were Student's t-statistic, both paired and unpaired comparisons being undertaken for all comparable samples, with the .05 level serving as the criterion for significant difference (cf. the introductory account in Gary Smith, Statistical Reasoning (Boston: Allyn and Bacon, 1985), 305-7, 347-48).

28Omstein, Gamelan Gong Kebjar: The Development of a Balinese Musical Tradition, 90-91.

29Hood, "Sléndro and Pélog Redefined." The Pliatan ensemble described by Ornstein is recorded on Anak Agung Gde Mandera, dir., Dancers of Bali (Columbia Special Products, CML 4618). Mantle Hood, Tjokorda Mas, and Wajan Gandera, dirs., The Exotic Sounds of Bali (Columbia Masterworks, Stereo MS 6445, Monaural ML 5845) contains recordings of Hood's gender wayang quartet and the UCLA kebyar ensemble.

30Hood, "Sléndro and Pélog Redefined," 31-32. 
Interesting in light of the present study and the investigations of Hood and Ornstein is the statement made long ago by psychoacousticians Stanley Smith Stevens and Hallowell Davis that "at the rate of about 6 or 7 beats per second, where the second stage [of beating] begins, the smooth rise and fall in loudness [produced by smaller differences in cps] vanishes and each beat appears as a single impulse." 31 Stevens and Davis are careful to point out that "the boundaries between these stages [i.e., of beating] are not sharp, but the character of the sensation within each stage is quite distinct." 32 These psychoacoustical observations and others reported below can be easily and precisely verified by writing simple programmes using, for example, the WAVE, SOUND, SOUND WAIT, and SOUND RESUME commands in Microsoft Basic on a Macintosh computer.

The oft-noted "shimmer" in Balinese gamelan music can be traced, in the Pliatan, Universe, and UCLA ensembles, as well as in Hood's wayang quartet, to the fact that the paired, penjoreg-producing instruments overwhelmingly tend to produce beating rates within the range at which impulse-beating rather than smooth loudness-undulation occurs, that is, approximately 6 or $7 \mathrm{cps}$ or a little faster. Stevens and Davis also observe that impulse-beating gives way to perceived "roughness" at about $20 \mathrm{cps}^{33}$ Their Figure $100^{34}$ strongly suggests that such roughness peaks at approximately $50 \mathrm{cps}$ throughout the range of frequencies relevant to the Universe gamelan and the ensembles measured by Hood and Ornstein. Noteworthy in this respect is that even the very highest penjoreg-rates reported precisely for the Universe, Pliatan, and UCLA ensembles $(10.2,10.5$, and $6.75 \mathrm{cps}$, respectively) are quite far from the 20-cps rate at which roughness generally begins to be prominent. What seems to have been aimed at in all the ensembles is impulse-beating at fairly low rates (i.e., about 7 impulses per second) within individual penjoreg-pairs.

More than half a century ago, Stevens and Davis also noted that "at this same rate of modulation [i.e., 6 or $7 \mathrm{cps}$ ], the pitch of a vibrato ceases to rise and fall, as it does at lower rates," 35 and music psychologist Carl Seashore reported that large samples of vocal and violin vibratos produced by Western concert-music performers ranged from 5.9 to $7.8 \mathrm{cps}$, and from 5 to $10 \mathrm{cps}$, respectively. ${ }^{36}$ Both of these ranges are close to the beating rates found in the orchestras considered here. Just as vibrato can be regarded as adding "shimmer" to a voice, the penjoreg beating measured in the present study can be understood as adding a "vocal" element to the sounds of keyed metallophones.

In contrast to the paired, penjoreg-producing instruments, unpaired, non-penjoreg instruments (i.e., the residual pairs of penjoreg-pairing, which include genders 1 and 3, and 2 and 4, as well as kantils 1 and 3, and 2 and 4) tend to

31 Stevens and Davis, Hearing, 242.

32Loc. cit.

33 Ibid., 245.

34Loc. cit.

35Ibid., 242.

36Carl Emil Seashore, Psychology of Music (New York: McGraw-Hill, 1938; repr., New York: Dover, 1967), 35. 
produce smooth undulation, ranging up to about $4 \mathrm{cps}$. Of such relatively slow beats, Stevens and Davis say: "The first stage begins at an indefinitely low rate. The slowest rate of beating that can be detected is probably determined only by the patience of the listener. Wever reports listening to beats as slow as one in two minutes.... When the rate is increased to the vicinity of 2 or 3 beats per second, we find that the waxing and waning of loudness is very prominent. This is the rate at which beats are most easily detected." 37 It would seem that, on the whole, slow, smooth loudness undulation was aimed at in the tuning of the residual gender pairs, whereas much faster, more prominent undulation was sought in the residual pairs of kantils.

The distinction between the residual genders' and kantils' rates of undulation results in the kantils being isolated timbrally (as well as registrally) from the genders. To be sure, even the relatively fast undulation of the residual kantils can be quite difficult to "hear out" in actual performance. For instance, Colin McPhee indicated that the piece from which Examples 1 and 2 are drawn was traditionally performed at the rate of $\mathbf{5 8}$ half notes per minute. Its longest upper tones correspond to a quarter note in the score and are slightly less than half a second in length. Within a single tone, there is barely time for two fast-undulation cycles, at the highest, $4.3 \mathrm{cps}$ rate. Indeed, the bulk of the piece's upper part consists of eighth notes in the kantils, and these allow for approximately only a single undulation or less.

McPhee specifies that in interlocking, kotekan passages, gender 1 and kantil 1 generally play $G$ and $A$, genders 2 and 4 and kantils 2 and 4 play $B$ and $D$. In his account of the broken kotekan style, gender 3 and kantil 3 play $G$ and A, whereas, in woven kotekan, these two (kilitan) instruments play lines that weave together all four tones. ${ }^{38}$ In this sort of traditional performance practice, only if gender 3 and kantil 3 play an interweaving line is there any impulse-beating in kotekan passages, and such penjoreg-beating is restricted to B and D. However, this reduction in unison-beating rates within the gender and kantil groups is compensated for partially by octave-beating between genders and kantils (discussed below). In another version of kotekan performance practice, genders and kantils 1 and 2 play $G$ and $A$, and 3 and 4 play B and D in the broken style, whereas, in the woven style, pair 2 or 3 plays an interweaving line. Such a practice ensures constant penjoreg-beating for all tones in all playing-styles, as does, to a lesser degree, the pairing of instruments 1 and 4 , and 2 and 3 in broken kotekan and any of its corresponding woven practices. Despite the authority of McPhee's account, one must acknowledge that, in the absence of penjoreg-beating, slow undulation at the rates found in the Universe gamelan yields, in a $1 / 3-2 / 4$ kotekan pairing, a great reduction in overall volume and brilliance within individual unison-pairs.

In general, "the pitch" of a group of tones tuned close to a single frequency tends to correspond to their average frequency. ${ }^{39}$ For example, the G5's pro-

37 Stevens and Davis, Hearing, 242. Their reference is to E.G. Wever, "Beats and Related Phenomena Resulting from the Simultaneous Sounding of Two Tones," Psychological Review 36 (1929): 402-523.

38McPhee, Music in Bali, 254-55.

$39 \mathrm{Cf}$. Stevens and Davis, Hearing, 243. 
duced by kantils 1 and 2 have frequencies of 800.5 and $807.0 \mathrm{cps}$, respectively. As a simultaneous pair, these G5's tend to be heard as matching, in pitch, a tone of $((800.5+807.0) / 2 \Rightarrow) 803.75 \mathrm{cps}$. However, this is only a tendency. As in all instances of such matching, one is concerned with probabilities rather than determinate values. $803.75 \mathrm{cps}$ is merely an estimate of the most probable single frequency which such a pair would match.

Whereas Western music theory has reified pitch by referring loosely to "the pitch" of one or more tones, determinations of such a value are always relational and probabilistic, and always involve an arbitrary benchmark (e.g., a sine-tone of $1000 \mathrm{cps}$ at 40,60 , or 80 , etc. decibels, because pitch can vary to a certain extent with intensity). Nonetheless, the undulation- and impulse-unisons in the Universe gamelan are sufficiently narrow to provide a basis for estimating, within rather small ranges and with quite high probabilities, intervallic relations between pairs of such unisons (e.g., in order to determine whether G3-A3 in the jegogans matches G4-A4 in the genders: see Figure $3 \mathrm{~b}$ below).

\section{Octaves}

In the mid-sixties, Hood drew attention to the fact that octaves in Indonesian ensembles tend not to accord very closely to the 2:1 (i.e., 1200 cents) proportion of historical Western (and many non-Western) tuning theories. ${ }^{40} \mathrm{Hood}$ wrote of "stretched" and "compressed" octaves, and since then, careful studies of gamelan tuning have borne out his observations.

In general, the octaves of the Universe gamelan are "stretched" (i.e., larger than 1200 cents) rather than "compressed" (i.e., smaller than 1200 cents). This observation coincides with Dowling's account of octaves on fixed-pitch instruments of four gamelan ensembles (two slendro and two pelog), measured by Hood and by Wasisto Surjodiningrat, P.J. Sudarjana, and A. Susanto, as well as octaves on a Burmese harp. ${ }^{41}$ Similarly strong tendencies for octaves to be stretched rather than compressed appear in my analyses of 37 pelog ensembles and in Wim van Zanten's account of variable-frequency instruments in West Java. ${ }^{42}$

Dowling astutely pointed out that the stretched octaves he found in gamelan ensembles corresponded to the octaves produced by Westerners in experimental studies undertaken by music psychologists W.D. Ward and K. Walliser and that similar findings obtained in a study of Westerners by J.E.F. Sundberg and J.

40Hood, "Sléndro and Pélog Redefined," 31-32.

41 W. Jay Dowling, "Scale and Contour: Two Components of a Theory of Memory for Melodies," Psychological Review 85, no. 4 (1978): 341-54, esp. 344-45. The gamelan measurements were based on Hood, "Sléndro and Pélog Redefined," and Wasisto Surjodiningrat, P.J. Sudarjana, and A. Susanto, Tone Measurements on Outstanding Javanese Gamelans in Jogjakarta and Surakarta., 1st ed. (in Indonesian, 1969; 2nd rev. ed., Jogjakarta: Gadjah Mada University Press, 1972); the harp measurements presumably correspond to the equiheptatonic tuning described in Judith Becker, "Anatomy of a Mode," Ethnomusicology 13, no. 2 (1969): 267-79, esp. 270-71.

42Jay Rahn, "Javanese Pelog Tunings Reconsidered," Yearbook of the International Folk Music Council 10 (1978): 69-82, and "Working with Found Data: Indonesian Pélog Tunings," 40pp. unpub. ms. (Toronto: the author, 1980; rev. 1993); Wim van Zanten, "The Tone Material of the Kacapi in Tembang Sunda in West Java," Ethnomusicology 30, no. 1 (1986): 84-112. 
Lindqvist as well as in a study of non-Westerners by E.M. Burns (1974), the latter replicating Ward's much earlier (1954) experimental method. Dowling also drew attention to the study by D.W. Martin and Ward (1961), where it had been shown that the octaves produced by Western piano tuners tend to be larger than 1200 cents, especially at the lowest and highest frequencies. ${ }^{43}$

The origin of stretched piano-octaves has been traced to the stiff lower- and upper-most strings of pianos, which, on being struck, behave like individual chimes rather than strings. The spectra of chime-like tones are not harmonic, in the sense of not corresponding very closely to frequencies that would be predicted by the overtone series. ${ }^{44}$ However, non-fundamental portions of the spectra produced by the keyed metallophones considered here are quite evanescent and do not exhibit clear patterns beyond basic low/high and early/late distinctions that correspond largely to a thung/ping dichotomy. Reasons for stretching or compression in the Universe gamelan's octaves have to be sought elsewhere.

In order to describe the stretching of octaves in the Indonesian tone measurements he had gathered, Dowling suggested, as an estimate, the average octaveratio $2.02 / 1$ (=1218 cents), which he advanced in contrast to $2.00 / 1 \quad=1200$ cents). ${ }^{45} \mathrm{~A}$ more recent study by Dowling and Dane Harwood emphasizes that there might be considerable variation in the sizes of octaves in individual ensembles. ${ }^{46}$ In the Universe gamelan, the octaves between pairs of beating or undulating tones (cf. Fig. 3b) average 1243 cents $(=2.05 / 1)$ irrespective of possible playing style. The sizes of the octaves that contribute to each of the three averages for the Universe gamelan are, as individual aggregates, significantly larger than 1200 cents, and do not differ significantly from one another. Moreover, the jegogan-gender and gender-kantil octaves do not differ significantly from each other in any of the playing styles, even though in each style the lower octaves average about 1256 cents, and the upper, approximately 1230 . As in the instance of unisons, listeners with differing acuity might make more or fewer distinctions between octaves than are conveyed by assessments of statistical significance.

An important element in the (admittedly, non-significant) difference between upper and lower octave-sizes is the narrow gender-kantil G-octave that ranges from 1195 to 1183 cents, depending on the playing style. The data that Ward and Walliser provide for sine-tone octaves similarly do not feature absolutely uniform octave-sizes. ${ }^{47}$ Instead, the amounts, in cents, by which the top tones

43W.D. Ward, "Subjective Musical Pitch," Journal of the Acoustical Society of America 26 (1954): 369-80; K. Walliser, "Über die Speizung von empfundenen Intervallen gegenüber mathematisch harmonischen Intervallen bei Sinustönen," Frequenz 23 (1969): 139-43; J.E.F. Sundberg and J. Lindqvist, "Musical Octaves and Pitch," Journal of the Acoustical Society of America 54 (1973): 922-29; Burns, "Octave Adjustment by Non-Western Musicians"; Martin and Ward, "Subjective Evaluation of Musical Scale Temperament in Pianos."

44 Cf., for example, John R. Pierce, The Science of Musical Sound (New York: Scientific American Books, 1983), 181-83.

45Dowling, "Scale and Contour," 341-54.

46Dowling and Harwood, Music Cognition, 101-4.

47 Cf. ibid. 
of the octaves they examined exceed 1200 cents increase slowly and steadily up to $600 \mathrm{cps}$ (i.e., approximately, up to the highest of Universe's jegogan-gender octaves), level out between 600 and $800 \mathrm{cps}$ (i.e., between the jegogan-gender and gender-kantil octaves), increase quite quickly between 800 and $1000 \mathrm{cps}$ (i.e., around the gender-kantil G- and A-octave), resume a slower rate of increase between 1000 and $1800 \mathrm{cps}$ (i.e., through and beyond the B- and D-octave of the genders and kantils), and thereupon increase relatively steeply again. Not only is there variation in these average sizes, there is as well no simple mathematical (e.g., logarithmic or exponential) function that would capture this particular configuration of connections between octave-sizes (expressed in cents) and the absolute frequencies at which these octaves are located (expressed in cps).

There is no uniformity in sine-tone octave-sizes from one study to another that would suggest that sine-tone octaves "ideally" have such a single value as 2.02 or 2.05 . In his original study, Ward emphasized that preferred sizes of sine-tone octaves differed somewhat from one person to another. ${ }^{48}$ Nonetheless, octave-stretching, of whatever size and whether in Western and non-Western tunings or in studies of subjective interval-size East and West, constitute a compelling regularity in the literature on octaves. K. Ohgushi explains, in terms of temporal aspects of neurophysiology, the tendency of sine-tone octaves to be stretched, and admits extra, spatial (or "place") factors in accounting for complex-tone octaves. ${ }^{49}$ Nonetheless, the narrow gender-kantil G-octaves that appear in each traditional playing style of the Universe gamelan indicate that stretched octaves, even between the sine-tone-like spectra of the ensemble's metallophones, constitute only a tendency, not a deterministic "law."

Although the narrowness produced by the kantils' G's is expressible in terms of frequency ratios or cents, its full significance can be conveyed only in terms of absolute frequency differences or cps. In normal playing, the average frequencies of the genders' and kantils' G's are $406.4 \mathrm{cps}$ and $804.6 \mathrm{cps}$, respectively. These values are not mere statistical artefacts; instead, they correspond quite closely to the pitches that tend to perceived when the instruments play in ensemble. Within pairs of tones forming a unison, both at the top and bottom of this octave, there is penjoreg beating. Moreover, between each pair of tones that consists of one at the top and one at the bottom, there is also beating by virtue of aural or subjective harmonics.

In their classic account of music psychology, Stevens and Davis report that “just as two primary tones may produce beats, so may any of the harmonics of these tones, regardless of how the harmonics are generated. Particularly interesting is the possibility of hearing beats between various harmonics of two tones when the harmonics are generated in the ear" (my emphasis). ${ }^{50}$ Any pure, sine tone having a frequency of $x$ cps (including the fundamental of a complex tone) generates, in the ear, its own aural harmonics, having frequencies of $2 x, 3 x, 4 x$,

48Ward, "Subjective Musical Pitch."

49K. Ohgushi, "The Origin of Tonality and a Possible Explanation of the Octave Enlargement Phenomenon," Journal of the Acoustical Society of America 73 (1983): 1694-1700.

50Stevens and Davis, Hearing, 244. 
$5 \mathrm{x}$, etc. cps, and having, respectively, intensities that tend to decline, even though nothing in the actual, physical sound-wave itself need correspond to these higher frequencies. ${ }^{51}$

The perceptual reality of aural harmonics can be verified by the method of so-called "best beats." Listeners readily identify tones that produce the most salient beating with the aural harmonics of sine-tones having lower frequencies. The effect of an "actual" wave being heard as beating with a "non-existent" component of an actual, but much lower, wave is an especially prominent product of sine tones (or fundamentals) having low frequency and/or high intensity. For the frequencies involved in such octave effects in the Universe gamelan, fundamentals only have to be sounded at sensation-levels ranging from about 25 decibels (i.e., above threshold) for the lowest jegogan tones to about 45 for the highest tones of the genders in order to produce the relevant, first-octave aural harmonics in audible form. ${ }^{52}$ These minimal sensation-levels lie well within or below the very softest levels of musical performance. ${ }^{53}$

The ear's production of extra, aural harmonics in response to impinging soundwaves has been likened to the non-linear behaviour of cheap sound systems, which similarly add information to incoming signals. The actual energy involved in aural harmonics has been located, by means of cochlear microphonics, at specific places on the ears of such animals as cats and guinea pigs, which are morphologically similar in this regard to humans. By means of amplification, one can "listen" to the energy comprised in the aural harmonics produced by such animals' ears. In this way, it has long been established that aural octaveharmonics, on which depend the effects of beating considered here, are fully objective, despite earlier writers' use of the term "subjective" as a synonym for "aural" (i.e., "of the ear" as distinguished from "of the soundwave"). ${ }^{54}$

For the narrow gender-kantil G-octave, beating is to be heard not only between tones at the top and at the bottom, but also between actual, physical (as distinguished from physiological) tones at the top and aural, physiological harmonics of actual tones at the bottom. This effect is easily replicated by means of Microsoft Basic for the Macintosh computer. For the gender-kantil G's, the aural-harmonic effect yields, on average, a beating rate of 2(406.4)-804.6 $=-8.2$ cps between the fundamentals of the upper tones and the first overtones (or second partials) of the lower. This average (negative) $-8.2 \mathrm{cps}$ rate for octavebeating among the gender-kantil G's corresponds to impulse-, penjoreg-beating between fundamentals that form "actual" unisons in the ensemble. Around this average rate, the four gender and four kantil versions of $G$ produce a rich rhythm, comprising fully twenty-eight undulation and beating cycles that sound simultaneously during ordinary playing $\left({ }_{4} \mathrm{C}_{2}+{ }_{4} \mathrm{C}_{2}+4^{2}=6+6+16\right)$, and, in kotekan performance, at least six such cycles. In sum, although the gender-kantil

51 Ibid., $184-87$.

52Ibid., 110-11, 186-87.

53Cf. Pierce, The Science of Musical Sound, 106-27, and Harvey Fletcher, Speech and Hearing in Human Communication (New York: Van Nostrand, 1953; repr. Jericho, N.Y.: Acoustical Society of America, 1995), 103-5, 127-36.

54Cf. Stevens and Davis, Hearing, 187-90. 
G-octave is exceptionally narrow, it systematically preserves the absolute profile of penjoreg-beating and both slow and fast undulation characteristic of the ensemble in the normal style of traditional performance. By contrast, in McPhee's account of broken kotekan, penjoreg beating disappears from these G's, though the distinction between slow and fast undulation persists. In all possible styles of woven kotekan, all three kinds of unison beating are maintained.

Stevens and Davis indicate that beyond undulation and beating effects, there is perceived interaction between the second partials and fundamentals of tones forming, respectively, the lower and upper portions of octaves, even if the "subjective" and objective components differ in frequency by as much as (a) $120 \mathrm{cps}$ at a frequency of $200 \mathrm{cps}$, (b) $160 \mathrm{cps}$ at $800 \mathrm{cps}$ (i.e., around the gender-kantil G), or (c) $200 \mathrm{cps}$ at $3200 \mathrm{cps}$. The perceived interaction between such waves is characterized as roughness (cf. the account of unisons, above) and peaks at about $50 \mathrm{cps}$ for all three frequencies. ${ }^{55}$ These frequencies correspond quite closely, as a group, to the overall range within which appear the relevant (i.e., second and first) partials of all octaves in the Universe gamelan. Although there is considerable divergence from the $2 / 1$ ratio among these octaves, their relevant partials differ by much less than the boundary amounts just specified (i.e., $120 \mathrm{cps}$ at $200 \mathrm{cps}$, etc.) and produce, at most, rather slow rates of roughness.

The fastest, upper-boundary values for roughness correspond to the critical band in music psychology (a concept that also entails effects of masking, involving, for example, white noise, which are not relevant here). The critical band is defined, like several other psychoacoustical boundaries, in terms of very unlikely responses (e.g., the perception of roughness between sine tones of 200 cps and $320 \mathrm{cps}$, for which the probability is very low) rather than responses of high probability (e.g., a roughness perception between sine tones of $200 \mathrm{cps}$ and $250 \mathrm{cps}$ ). Estimates of the critical band's extent at a given frequency differ from study to study). ${ }^{56}$ Nonetheless, all octaves of the Universe gamelan correspond to frequency differences well within boundaries that have been determined experimentally. Further, such experimental determinations, despite discrepancies among them, point quite uniformly to the fact that the critical band corresponds, on the basilar membrane, to a quite constant physical distance or number of receptor cells that service a particular continuous region. ${ }^{57}$ Effects that directly involve the critical band correspond to physiological or neurological universals within the human species, as do effects of beating or aural harmonics.

For the Universe gamelan's octaves, differences of approximately a quarter to a half the critical band result in a perception that the partials involved are distinct from each other in pitch..$^{58}$ As is true of other perceptual thresholds, this

55Ibid., 245-47.

$56 \mathrm{Cf}$., for instance, E. Zwicker, G. Flottorp, and S.S. Stevens, "Critical Bandwidth in Loudness Summation," Journal of the Acoustical Society of America 29 (1957): 548-57.

57E.g., Juan G. Roederer, Introduction to the Physics and Psychophysics of Music, 2nd ed. (New York: Springer-Verlag, 1975), 26-33.

58Cf. R. Plomp, "The Ear as a Frequency Analyser," Journal of the Acoustical Society of America 
boundary (i.e., between distinctness and non-distinctness of objective and aural partials in tones with fundamentals that are fairly close to the $2 / 1$ ratio) is probabilistic and depends on the method or benchmark employed in its determination. Nonetheless, frequency differences between relevant partials of the Universe's octaves are, without exception, smaller than the amounts generally required for this sort of difference to be prominent.

The essence of the sort of octave distinctness considered here (and avoided in the Universe gamelan) is not merely that there are two distinct components to the octave, upper and lower. Such distinct components can be perceived quite easily even if fundamentals form a virtually perfect $2 / 1$ ratio. Instead, the effect consists of the objective upper fundamental being heard as producing clear "out-of-tune-ness" with the lower fundamental, an effect mediated by the lower fundamental's first (subjective) overtone and accompanied by roughness. Lower speeds of roughness do not give rise to the effect, which, if reached by degrees in a computer-assisted replication with sine-tones, is heard as a characteristic that has been added to the overall effect of a pair of tones.

For no octaves in the Universe gamelan is the effect of out-of-tune-ness between octave fundamentals prominent. Instead, upper-octave fundamentals produce effects of undulation, beating, or fairly slow roughness with the first aural overtones of lower-octave fundamentals. Except for the gender-kantil G's, from the lowest, jegogan-gender octaves to the highest, gender-kantil octaves, the widest octave-intervals of the eight $(=2 \times 4)$ or sixteen $(=4 \times 4)$ at each individual pitch (G, A, B, and D) exceed the $2 / 1$ ratio by amounts that range approximately from zero to $35 \mathrm{cps}$, depending on the playing style. The impacts of the greatest differences are diminished in normal playing by highly prominent undulation and impulse beating at the top and bottom of each octave. As well, the relatively slow rates of octave-beating in the ensemble tend to compress the perceived sizes of the octaves in which they occur. Within this pattern, the gender-kantil G-octave is exceptional. In normal playing, its average size is approximately $8 \mathrm{cps}$ smaller than the $2 / 1,1200$-cent value and comprises what might be called "negative" impulse beating; in both broken and woven kotekan (of all varieties), this G-octave yields negative undulation.

As a substitute for the idea that the octave "is" an interval with a frequency ratio of $2 / 1$ (or 2.02/1,2.05/1, etc), one can offer the notion of octaves as non-unison tone-pairs which feature maximal probability of interaction between their partials. That is, the octave is basically a timbral, not an intervallic, entity. The human voice and such instruments as the violin produce tones with spectra that are very close to perfect in harmonicity. Notwithstanding the effects of vowel formants in singing, the greatest amounts of their energy are concentrated quite close to frequencies of $x, 2 x, 3 x$, etc cps (where $x$ cps is the fundamental). Except for unisons, no pairs of such harmonically structured tones produce greater coincidence or overlap of partials than tones whose frequencies are fairly

36 (1964): 1628-36, and "Detectability Threshold for Combination Tones," Journal of the Acoustical Society of America 37 (1965): 1110; and Roederer, Introduction to the Physics and Psychophysics of Music, 26-33. 
close to $2 / 1$. The same situation holds for the aural, subjective harmonics that arise from sine-tones. In general, for a tone having "subjective" or "objective" spectral components comprising $\mathrm{x}, 2 \mathrm{x}, 3 \mathrm{x}$, etc. cps, the greatest possible coincidence or overlap, beyond the unison, arises from its interaction with a tone that has as its partials $2 \mathrm{x}, 4 \mathrm{x}, 6 \mathrm{x}$, etc. cps-that is, an octave. ${ }^{59}$

Notions of overlap and interaction are to be emphasized, as is the idea of being "fairly close" because, in a certain region of the basilar membrane, such an "interactive" octave might comprise, among its components, tones separated by such a large ratio as, say, 1309 cents (which, in fact, appears between the G's of jegogan 1 and gender 4 ).

Tuning theories in various cultures have tended to elevate the $2 / 1$ ratio to an extremely high status (e.g., the "perfection" of the $2 / 1$ octave in medieval formulations within the Aristotelian tradition). Such high status comprises an ideal that is, in principle, if only because of Uncertainty, absolutely unattainable in practice. Theories based on ideal octave-sizes have exalted, at least implicitly, maximal coincidence of partials over interaction or overlap. By contrast, Indonesian tuners have sought varied forms of spectral interaction that result from the overlap of large amounts of energy on the basilar membrane and comprise (short of pronounced out-of-tune-ness within octaves) not only beating but also roughness in varying degrees. An inclusive definition of the octave acknowledges such possibilities for interaction and relegates the ideal, $2 / 1$ ratio to the status of an extremely special case or speculative construct. Moreover, as is shown in further detail presently, the enormous variety of spectral interaction one can observe in Indonesian music seems to have been structured very carefully and deliberately by traditional tuners.

Hood cites a statement of Balinese musician Tjokorda Mas that octaves in different registers (e.g., in the present instance, in the jegogan-gender register and in the gender-kantil register) tend to differ in size by amounts corresponding to a half or whole penjoreg. ${ }^{60}$ With penjoregs (p) set at $7 \mathrm{cps}$, the following, schematic model fits the data of average unison- and octave-interaction in the Universe gamelan quite closely (all quantities are approximate and expressed in cps):
D $d$ to $d+p$
$2 \mathrm{~d}+3.5 \mathrm{p}$ to $2 \mathrm{~d}+4.5 \mathrm{p}$
$4 d+12.5 p$ to $4 d+13 p$
B $\quad b$ to $b+p$
$2 \mathrm{~b}+2.5 \mathrm{p}$ to $2 \mathrm{~b}+3.5 \mathrm{p}$
$4 \mathrm{~b}+9 \mathrm{p}$ to $4 \mathrm{~b}+10 \mathrm{p}$
A $a$ to $a+p$
$2 a+2 p$ to $2 a+3 p$
G $g$ to $g+p$
$2 g+2.5 p$ to $2 g+3.5 p$
$4 a+8 p$ to $4 a+9 p$
$4 g+4.5 p$ to $4 g+5.5 p$

The gist of Mas's account seems vindicated by this model (which can be checked in detail against the raw cps numbers provided in Figure 3a, above). The penjoreg and half-penjoreg appear to constitute coherent units (or increments) with regard to octave compression (cf. the gender-kantil G's) and stretching (all the other octaves).

59 Cf. H.R. Blackwell and H. Schlosberg, "Octave Generalization, Pitch Discrimination and Loudness Threshold in the White Rat," Journal of Experimental Psychology 33 (1943): 407-19, esp. 407.

$60 \mathrm{Hood}$, "Sléndro and Pélog Redefined," 32. One should note that although Mas was referring to kebyar, not angklung, the two sorts of ensemble are highly similar in the respects that are relevant here. 
For the Universe gamelan, the process of tuning generally involves avoiding $2 / 1$ octave duplication by at least half a penjoreg. This process can be regarded, in turn, as a sub-process of a larger, "spiral" process. From low to high, octaves tend to become larger not merely by virtue of cumulative stretching within the context of a relatively constant penjoreg size. For example, the gender B of $2 b+2.5 p / 2 b+3.5 p$ cps comprises an octave stretching of $2.5 p / 1.5 p$ in comparison with the jegogan $B$ of $b / b+p$ cps, with which $2 b / 2 b+2 p$ cps would form a perfect $2 / 1$ ratio. If the gender $B$ had been paired with a kantil $B$ of $4 b+6 p / 4 b+7 p$, another slight stretching of the octave (as compared with the aural harmonics $4 b+5 p / 4 b+7 p c p s$ ) would have resulted. One finds, instead, that the kantil B has average frequencies of $4 b+9 p / 4 b+10 p$ cps. This overall tendency toward increased stretching is quite consistent and gradual within each pitch class from its bottom to top register (i.e., reading left to right across each column in the above figure), and within each register from its bottom to top tone (i.e., from bottom to top within each row)-the principal exception being, again, the gender-kantil G's.

In attempting to discern overall patterns in the widespread practice of octavestretching, investigators have provided summary statements, graphs, and mathematical models. The sizes of octaves, expressed as frequency-ratios (i.e., in cents), are described as displaying various tendencies from low to high frequencies. Depending on the formulation, octaves are shown as remaining the same, increasing, alternating between similarity in size and augmentation, or (as here) diminishing at a single pitch class within a statistically non-significant decline. Common to such studies of octave-stretching is the finding, implicit or explicit, that octaves generally increase in size relative to the $2 / 1$ ratio, if these differences are expressed in cps. Nonetheless, as emphasized above, the gender-kantil G's, though highly exceptional, indicate that no deterministic "law" of octavestretching is involved in the phenomenon, only a tendency, or probability. Deviations from the $2 / 1$ ratio, even expressed in cps, might rise or fall-even into the negative values of octave-compression.

In the middle range of hearing, where the functional intervals of music tend to be located, there appear to be certain constraints on octave-size which, nonetheless, allow for a wide variety of realizations. These constraints seem to involve (a) ensuring spectral interaction, whether in the form of sheer (but, in principle, unattainable) coincidence, undulation, beating, or roughness), (b) avoiding out-of-tuneness between upper fundamentals and the (aural) first overtones of lower fundamentals, and (c) varying octave-sizes in a gradual, rather than sudden, manner.

"Naturally occurring" octaves, as distinguished from electronically contrived octaves, appear to have been subject to the sorts of constraints just listed. ${ }^{61}$ The products of speculative, numerically formulated, historical tuning theories in various cultures constitute a very special case of these constraints, and modern experiments with electronic sounds ${ }^{62}$ have explored extreme breaches. Histori-

61Cf. Pierce, The Science of Musical Sound, Exx. 2.1-2.5, 224-26 for recorded examples of synthesized spectra and intervals that feature stretching.

62E.g., F.H. Slaymaker, "Chords from Tones Having Stretched Partials," Journal of the Acoustical Society of America 47 (1970): 169-71. 
cal theories have sought, at least implicitly, sheer coincidence. If realized in the harmonic spectra produced by both voices and the principal instruments of the cultures in which they have arisen, such ideal formulations would guarantee absolute in-tune-ness and (trivially) gradual change (i.e., no change at all) from low to high. By contrast, contemporary experiments in sound synthesis have investigated large deviations from coincidence and/or harmonicity. In the middle of this continuum can be located much of the actual music of various cultures. In such music, acoustic instruments and voices with spectra that are very close to harmonic in (objective or subjective) structure and octaves deviating quite a bit from the $2 / 1$ ratio have been frequent.

Arguably, the octave and unison are intervals principally to be explained in spectral, timbral terms rather than frequency ratios. Even in the instance of monophony (e.g., the solo introductions to angklung pieces), the unison can, in principle, yield timbral relations relevant to the octave by way of aural harmonics. Of these harmonics, the first is both the most intense and extremely close to twice the frequency of the fundamental that generates it, notwithstanding necessary side bands in both. The $2 / 1$ ratio provides ultimately an approximate numerical model of actual qualitative effects. Although spectral, timbral relations of the sort just considered correspond most directly to differences in frequency (even when these are virtually null), tones forming an octave also can be heard intervallically in a manner that corresponds most directly to differences in frequency-ratio (measured in cents).

Quite plausibly, the origin of the octave as a highly privileged entity in musical cultures is to be explained by the quite intense spectral interactions that arise when people sing in octaves (e.g., adult males on the one hand, and adult females or children on the other). Adult male speech tends to be about an octave lower than the speech of adult females and children. ${ }^{63}$ The vocal cords of adult males tend to be about $3.6 \mathrm{~cm}$ long, as compared to those of adult females and children, which tend to be about $2.6 \mathrm{~cm}$ in length, yielding, all other factors being equal, vibration rates that are approximately $(3.6 / 2.6)^{2}=1.92$ as slow as those of children and female adults. ${ }^{64}$ In natural languages (including such tone languages as Mandarin and "stress-accent" languages like English), pitch relations (i.e., between fundamental frequencies) function significantly. ${ }^{65} \mathrm{Human}$ evolution might well have favoured the genes of children who, during early childhood, best acquired the pitch skills of their language group by matching, at the unison, the frequency-patterns of adult, generally female, relatives whose vocal cords tended to produce fundamental frequencies closest to their own, and secondarily, by matching, through spectral interaction at the octave, the frequency-patterns of other, generally male, adult relatives.

63 Alan Cruttenden, Intonation (Cambridge: Cambridge University Press, 1986).

64J.E. Gordon, Structures (London: Penguin, 1978), 116-17.

65Cruttenden, Intonation; cf., as well, Bryan Levman, "The Genesis of Music and Language," Ethnomusicology 36, no. 2 (1992): 147-70 on the evolutionary primacy of pitch relations in both speech and song. 
That those who produce lower frequencies might tend to form octaves with those having higher voices can be accounted for in terms of maximal spectral interaction beyond the unison, whether in speech or song. ${ }^{66}$ That octave-perception, as such, might be inherited, or even merely easily learned, is indicated by the observation of non-human species responding to octaves in a manner more similar to their responses to unisons than to other intervals that are closer in physical, acoustical size to octaves. ${ }^{67}$ The widespread organization of music according to structures that correspond directly to relations between frequency-ratios (rather than frequencies) could be a result of low voices duplicating, at the octave, frequency patterns in speech or song produced by high voices. According to this conjecture, the acts of people who produced, respectively and simultaneously, fundamentals of $x$ and $y \mathrm{cps}$, and (approximately) $2 x$ and $2 y$ cps, would be reinforced by spectral interaction. Such acts would not be merely acts of spectral-octave production but also acts of frequency-ratio matching, in the sense that sounds producing the ratio $x / y$ would be heard as matching sounds producing ratios very close to $2 x / 2 y$. The relations involved in spectral and frequency-ratio perception differ basically. Nevertheless, both could, in principle, be reinforced by the same, octave outcomes.

In accounting for spectral phenomena, cps form a relevant unit, whereas for intervallic phenomena cents are more appropriate. Such intervallic relations as "is heard as matching intervallically" or "is heard as being intervallically wider than" can arise on their own or in connection with octave phenomena. Experimental studies of interval perception have hinged on the distinction between mere intervals and functional intervals. Throughout the middle range of frequencies (where the bulk of information for speech and music is concentrated), the probabilistic boundaries between the frequency-ratio sizes of pairs of tone-pairs that are heard as matching and not matching remain quite constant. Nonetheless, for Westerners (especially those with musical training), the generally rather even increments according to which tone-pairs are matched or distinguished intervallically are significantly smaller if close to such ratios as 350 and 450 cents than they are around $300,400,500$, etc. cents. For example, tone-pairs of 340 and 360 cents are distinguished from each other with a much higher probability than are tone-pairs of 280 and 300,300 and 320, 380 and 400, and 400 and 420 cents. $^{68}$ Though little investigated outside the West, this aspect of intervallic perception appears not to be restricted to Euro-American music. ${ }^{69}$

66Cf. Jay Rahn, “Pélog Tunings, Continued," Ethnomusicology Research Digest 30 (1990).

67E.g., Blackwell and Schlosberg, "Octave Generalization."

68Cf. Burns and Ward, "Categorical Perception."

69Parallel findings arise in my recent comparative study of interval-labeling by eight Western music majors and the distinguished bi-musical Egyptian-Canadian performer/ethnomusicologist George Sawa, who has had extensive formal training in both European and Middle Eastern music. The findings corroborated the results for Westerners reported by such music psychologists as Burns and Ward, "Categorical Perception," and turned up, for Dr. Sawa, patterns at the levels of both a quarter and an eighth tone that corresponded quite closely to the students' responses at the single level of a semitone. Dr. Sawa regularly produces the eighth-tone interval (or "comma": ca. 25 cents) in his professional practice of playing the qanun. 


\section{Other Intervals}

In gamelan angklung, the most salient intervals other than the octave or unison are those that appear successively on individual instruments. Within each group of instruments (jegogans, genders, and kantils), the following melodic intervals are regularly used: G-A, A-B, B-D, G-B, A-D, and G-D (see Exx. 1 and 2). If one compares, in cents, all the G-A's with all the A-B's, B-D's and A-D's, all the A-B's with all the B-D's, all the B-D's with all the G-B's, and all the G-B's with all the A-D's, one finds a perfect coincidence with an important aspect of the tuning pattern for equally tempered anhemitonic (i.e., semitone-less) pentatonic. The G-A's as a group do not differ significantly from the A-B's; both are significantly smaller than the B-D's, which are significantly smaller than the G-B's, which, finally, are significantly smaller than the A-D's (and, of course, than the G-D's). This finding accords with the Western formulation of anhemitonic pentatonic, where G-A and A-B both comprise two semitones; B-D, three; G-B, four; A-D, five; and G-D, seven. By contrast, in equipentatonic slendro, G-A, A-B, and B-D would be the same in size (i.e., 1/5 octave), as would G-B and A-D ( $2 / 5$ octave). In other words, the Universe gamelan is much closer in its ordering of interval-sizes to anhemitonic pentatonic than to equipentatonic slendro.

In mathematics, an ordinal formulation is cast entirely in such phrases as "larger than," "smaller than," and "the same in size as." Such an account would not specify the amount by which B-D exceeds G-A and A-B, or G-B exceeds $B-D$, etc. Relative to a G-D interval set (arbitrarily, and only for the sake of illustration) at 700 cents, an ordinal anhemitonic pentatonic model would allow, in principle, for such extreme configurations for G-A, A-B and B-D as the following: 171,171 and 348 , and 234,234 , and 232 cents.

A fully numerical formulation of anhemitonic pentatonic would acknowledge that $B-D$ is one semitone larger than $G-A$ and $A-B, G-B$ is one semitone larger than B-D and two semitones larger than $G-A$ and $A-B$, and so forth. Nonetheless, one need not invoke specific numbers, as such, in order to capture the gist of the latter, numerical, semitone-based formulation. One can specify instead that the amount by which B-D exceeds both $\mathrm{G}-\mathrm{A}$ and $\mathrm{A}-\mathrm{B}$ matches the amounts by which $G-B$ exceeds $B-D$ and $A-D$ exceeds $G-B$, and that the amounts by which $G-A$ and $A-B$ exceed the unison, $G-B$ exceeds $G-A$ and A-B, A-D exceeds B-D, and G-D exceeds A-D all match each other. All of these relations among intervals, though not explicitly numerical, are more constrained than merely ordinal (i.e., greater-than, less-than, and matching) relations between intervals, and all hold statistically for the intervals produced in even the most extreme of the Universe gamelan's possible traditional performing styles (as summarized in Figure 3b).

Such comparisons involve mere interval-relations, rather than functional intervals connected directly in size to the octave. In a mere-interval comparison of the sort just outlined, G-D is not necessarily connected in size to the octave's size. Since only intervals other than an octave are being compared, G-D might, in principle, have any size that corresponds to seven twelfths of any feasible octave whatever. In order to assess interval sizes other than an octave in terms 
of actual octaves, which might differ considerably from 1200 cents, one has to provide room within a model for comparisons that take into account the sizes of actual octaves in assessing relations between other, non-octave intervals.

A comparison of interval sizes in the contrasting playing styles of Figure $3 \mathrm{~b}$ with ideal models of equipentatonic slendro and equally tempered anhemitonic pentatonic that allow the octave-size to "float," as it were, yields the general finding that equally tempered anhemitonic models specify interval-sizes that are uniformly much closer to the actual sizes found in the different performance styles than are equipentatonic, "pure slendro" models. Within this framework, the greatest deviations from anhemitonic pentatonic arise not only in the gender-kantil G's but also in the jegogans' $D$, which, in a functional formulation, is compared intervallically with all other tones in the ensemble, not just with tones in its own register. Both the slendro and pentatonic models yielded very high values for $R^{2}$, namely, 0.999 and 1.000 , respectively. The standard errors of the estimates, intercepts, and slopes were uniformly much smaller for comparisons of tunings in the various playing styles with pentatonic than with slendro models: 16 to 17 cents, 8 to 9 cents, and .005 (i.e., 6 cents) for pentatonic, in contrast to slendro: 35 to 38 cents, 18 to 20 cents, and .01 to .011 (i.e., 12 to 13 cents), respectively. For pentatonic, the estimates of the octave-sizes (i.e., based on the slopes) ranged from 1242 to 1240 cents, both of which were substantially closer to the actually observed average octave-sizes ( 1243 cents: see above) than are the slendro estimates, which ranged from 1254 to 1252 cents. The absolute sizes of the residuals for all the pentatonic models were significantly smaller than for the corresponding slendro models, but for both sorts of model, these deviations were substantially greater than zero cents. By far the greatest divergences of individual tones from the pentatonic models involved uniformly the gender and kantil G's (which ranged from 14 to 31 , and from 34 to 27 cents, above and below, the model values, respectively, cf. above) and the jegogan D's (which ranged from 24 to 26 cents below the model values); in this sense, all three tones in the various playing styles could be considered "errant" along the lines of Hood's formulation ("Sléndro and Pélog Redefined").

The range of possible models for angklung tunings is very large. Colin McPhee, using his "ear" rather than acoustical equipment, characterized various angklung tunings in terms of equal temperament. He arrived at the following results for various ensembles:70 E, F-sharp, A, B (Exx. 225-28, 230-32, and 235); F, G, A, B (Exx. 233-34); and F, G, A, C (Ex. 236) as well as A-flat, B-flat, C, E-flat (Ex. 237-39, 244-46, and 248-50). Evidently, relative to equal temperament, there is a wide variety of traditional tunings for the four-tone pieces of angklung. The following questions arise: What features are shared by all known angklung tunings? And how can one best characterize differences between tunings? Such questions are germane, because all traditional tunings for angklung are acknowledged within Balinese culture as belonging to a single general category, namely, slendro, and individual pieces are often played on ensembles having quite different versions of slendro tuning. As well, within

70McPhee, Music in Bali, 234-55. 
Indonesian musical culture, various individual tunings are regarded as having their own particular characteristics, as in Suweca's description of the Universe gamelan as having "a very sweet sound" (above), and as in Susilo's account of "embat" in Javanese tuning (above, as well).

\section{Individual Tunings and Tonal Systems in General}

Rendered in the ideal figures of equal temperament and relative to an octave, having, as its ideal size, 1200 cents, the angklung tunings implicit in McPhee's transcriptions are as follows (each transposed to begin on G, for the sake of comparison: the second of these corresponds to the ideal anhemitonic pentatonic interval-sizes that could be considered to underlie the Universe angklung):
G
200
200200
A B
C-sharp
[E]
[300)
(300]
(G) - cf. F G A B
G
A B D
200
200
300
(200]
[E] (G) - cf. F G A C; A-flat B-flat C E-flat
G ${ }_{200}{ }^{\text {A }}{ }_{300}{ }^{\text {C }} 200^{\text {D }}$
(200]
[E] (G) - cf. E F-sharp A B
[300)

Each of these ideal patterns can be regarded as constituting four of the five tones in a five-pitch-class cycle, where, in any pair of adjacent tones (e.g., G-A, A-B, and B-C-sharp, in the first pattern, above) the lower tone (e.g., G, A, B) is a major proportionate half-octave, and the upper (e.g., $A, B$, and $D$, respectively) a minor proportionate half-octave, below a third tone. Relative to an ideal octave of 1200 cents, major proportionate half-octaves range from 600 to 800 cents; minor, from 600 to 400 cents (i.e., from one-half to two-thirds, or from one-half to one-third such an octave). ${ }^{71}$ The fifth, "missing" tone (e.g., E, relative to $\mathrm{G}, \mathrm{A}, \mathrm{B}$, and $\mathrm{C}$-sharp or D) can be regarded as implied, rather than explicitly presented. ${ }^{72}$ In the above examples, $G$ is 600 cents below $C$-sharp and $A$ is 400 cents below C-sharp; $A$ is also 700 cents below [E] and B is 500 cents below [E]; and so forth.

The same situation holds for such patterns as the following (the first corresponding to an ideal version of equipentatonic, the second based on ideal unit-intervals of $133.3(=1200 / 9)$ cents in an equinonatonic framework (see below), the third comprising explicit semitones (G/A-flat and C/D-flat) in a non-pelog pattern, and the fourth an implicit semitone (D-sharp/E), also in a non-pelog pattern:

71 Cf. Jay Rahn, "Some Recurrent Features of Scales," In Theory Only 2, nos. 11/12 (1977): 43-52.

72 Cf. Jay Rahn, "Coordination of Interval Sizes in Seven-Tone Collections," Journal of Music Theory 35 , nos. $1 / 2$ (1991): 33-60, esp. 44-46. 


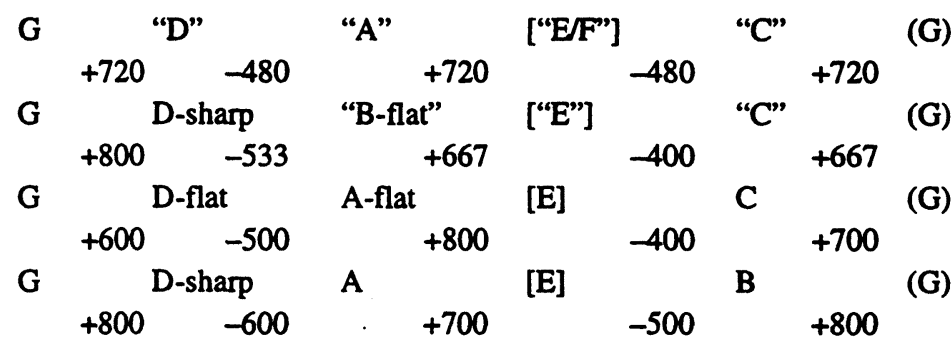

In this way, one can characterize determinately a broad range of four-tone configurations that would satisfy the fivefold cycle of half-octaves just outlined. Moreover, one can cite four-tone patterns that would falsify this cyclic arrangement (e.g., G, B-flat, C-sharp, E, and G, A-flat, B-flat, D-the latter comprising a semitone, G-A-flat, in a pelog pattern). The fivefold half-octave cycle serves to define five-tone (i.e., "pentatonic") scales in general, as distinguished from, for instance, seven-tone (i.e., "heptatonic") scales, and both sorts of scales from what might be termed "non-scales" (i.e., patterns which do not, in themselves, constitute a half-octave cycle). ${ }^{73}$ In this way, the great variety of observed tunings within slendro can be considered to form a unit or category that can be distinguished from other, non-slendro tunings and that, in principle, comprises (or "generates") infinitely more realizations than have been, or ever could be, examined in the manner undertaken here.

Functionally, each instance of slendro, however uneven its realization might be, can be regarded as comprising five scale degrees that match each other proportionately in size, relative to the octave and by way of the half-octave, which corresponds to +3 and -2 steps in the above illustrations. The "pure" form of slendro, with ideal scale degrees of 240 cents, is a special case where each interval of one scale degree (e.g., G-"A," above) matches every other one-degree interval precisely, e.g., "A"-“C," "C"-"D," and, if they are present, "D"-"E/F" and "E/F"-(G) above; each two-degree interval (e.g., G-"C," above) matches every other two-degree interval precisely, e.g., " $A$ "-"D," "C"-"E/F"where present-, "D"-(G), and where present "E/F"-(A); and so forth. In combination with this special character, such a "pure"-slendro configuration entails (a) no "differences" (i.e., there are no intervals that have the same size in scale degrees but differ in functional-interval size, e.g., as measured in ideal semitones or fifths of octaves), (b) no "ambiguities" (i.e., no intervals of the same functional-interval size that differ in scale-degree size), (c) no "contradictions" (i.e., no intervals, $x$ and $y$, such that $x$ is larger than $y$ in scale degrees but smaller than $y$ in functional-interval size), ${ }^{74}$ (d) no "disproportions" (i.e., no intervals $x$ and $y$ such that (i) $x$ is precisely half the size of $y$ in scale degrees, e.g., 1 as compared with 2 scale degrees, and 2 as compared with 4 , but (ii) $x$ is

73Cf. Rahn, "Coordination of Interval Sizes in Seven-Tone Collections," 51.

74Cf. Dowling and Harwood, Music Cognition, 100-1, and G.J. Balzano, "The Group-Theoretic Description of 12-fold and Microtonal Pitch Systems," Computer Music Journal 4, no. 4 (1980): 66-84, on the closely related notion of "coherence;" also Rahn, "Coordination" and John Clough and Jack Douthett, "Maximally Even Sets," Journal of Music Theory 35 (1991): 93-173. 
not proportionately half the functional-interval size of $y,{ }^{75}$ and (e) no incommensuracies (i.e., no intervals $x$ and $y$, such that $i$ ) $x$ differs from $y$ by certain amounts, $a$ and $b$, with regard to scale degrees and functional interval-size, respectively, and ii) there is no other interval, $z$, such that $y$ differs from $z$ by scale-degree and functional amounts, $a^{\prime}$ and $b^{\prime}$, which match $a$ and $b$, respectively).

Instances of anhemitonic pentatonic involve distinctions between sizes at a level of detail beyond the scale degree. Such configurations involve differences (cf. (a), above), but no ambiguities (b), no contradictions (c), no disproportions (d), and no incommensuracies (e). With regard to differences, G-A, A-B, and (where present) D-E are all one-degree intervals in the collection G, A, B, D, [E], (G), and match in functional-interval size at the level of the semitone; these intervals differ, at the semitone level, from the remaining one-degree intervals, B-D, and-if E were present-E-(G). Similarly, whereas A-D, B-[E], D-(G), and [E]-(A) comprise two scale degrees and match each other in semitone-size, each differs in semitone-size from the remaining two-degree interval, G-B. And so forth.

Finally, what might be termed "whole-tone" patterns (e.g., G, A, B, C-sharp, [E], and G, A, B, D-sharp, [E]) involve: (a) differences (compare G-A and A-B with B-D-sharp), and (b) ambiguities (for, in the collection G, A, B, C-sharp, [E], (G), the six-semitone interval G-C-sharp comprises three scale degrees whereas the six-semitone interval C-sharp-(G) comprises only two, and in the collection G, A, B, D-sharp, [E], (G), G-B comprises four semitones and two scale degrees, whereas B-D-sharp comprises four semitones and one scale degree, and so forth), but (c) no contradictions, (d) no disproportions, and (e) no incommensuracies. In sum, the perceptual "rewards" or "reinforcers" for hearing a level of detail or "grain" in addition to half-octave/scale-degree quantization are greater for anhemitonic than for "whole-tone" patterns.

Within the framework just outlined, the Universe angklung comprises a gradated hierarchy that includes the following features, which persist, wherever possible, in the various playing styles: (a) four tones from a five-tone half-octave/scale-degree cycle, within which one can distinguish the relative paucity of differences and general lack of ambiguities, contradictions, disproportions, and incommensuracies characteristic of anhemitonic pentatonic; (b) "errant tones" corresponding to the gender-kantil G's and jegogan D; (c) quite wide, but nonetheless interactive octaves that generally spiral upwards in increments corresponding to the penjoreg and half-penjoreg; (d) beating of ca. $7 \mathrm{cps}$ between instruments that form penjoreg-pairs; and (e) two rates of undulation, fast and slow, between the instruments of non-penjoreg pairs.

\section{Gamelan-Related Musicianship}

Gamelan instruction in North America typically has focused on skills in performance, improvisation, or composition. ${ }^{76}$ The hierarchical framework just

75Cf. Rahn, "Coordination of Interval Sizes in Seven-Tone Collections," 35-36, 53-56, and John Clough, "Diatonic Interval Cycles and Hierarchical Structure," Perspectives of New Music 32, no. 1 (1994), 228-53.

$76 \mathrm{Cf}$. the already classic accounts of Judith Becker, "One Perspective on Gamelan in North America," Asian Music 15 (1983): 82-90, and Sumarsam, "The Meaning of Gamelan Performance," Progress 
described indicates the possibility of introducing gamelan-related ear training systematically into a music curriculum. As indicated above, one can synthesize gamelan-like tones readily by means of such software as Microsoft Basic and Quick Basic. In principle, responses to such tones, alone and in combinations, can be differentially reinforced (or "rewarded") within such multimedia software as Hypercard. For example, responses corresponding to such words as "ping," "thung," "undulating," "beating," "rough," "faster," "flat," and "narrow" can be reinforced convergently for stimuli that are increasingly close to various benchmarks. In principle, as well, corresponding skills in tone production can be developed by differentially reinforcing vocal sounds within pre-established, generally diminishing boundaries of frequency or, for example, clicks on a screen that has been programmed to produce various sounds along a continuum.

At a "macro"-level of pitch structure, tones from actual instruments can, in principle, be sampled and entire works or sections synthesized according to various tunings. Skills that can be developed in this format include the recognition and characterization of errant tones relative to a given overall tuning and of shifts in unison and octave interaction patterns, for instance, at the beginning of a kotekan passage. At the level of scalar structure, students can learn to sing a given piece in, for example, G-A-B-D, G-A-B-C-sharp, etc. "tunings," just as, in Western musicianship training, they might learn to sing any particular diatonic melody in any of six or seven "modes" (i.e., relative to a single "tonal centre" or "finalis"), or undertake vocally certain basic transformations of atonal sets.

Working actively with traditional, non-written music of many sorts demands a perceptual and productive flexibility, which, in many ways, is the opposite of the highly convergent tuning skills sought often in the training of Western concert musicians. In principle, people can acquire productive and perceptual fluency in "de-tuning" and "re-tuning" with a view to international, "world musicianship" by means of voice- and computer-based instructional pedagogies.

\section{Tunings, Numbers, and Contemporary Music Theory}

Throughout the present study, and, to a large extent, throughout the existing literature on Indonesian tunings, formulations have been cast in specific numbers. However, music psychologist Juan Roederer emphasized two decades ago that whereas responses which are qualitative (e.g., indications that two stimuli match or not) or only minimally quantitative (e.g., indications that one stimulus is merely larger or smaller than another in some respect) are much more reliable than fully numerate responses (e.g., indications that the frequency of one tone is " $3 / 2$ " the frequency of another, or that one tone is " $1 / 2$ as high as" another, the latter as in the determination of the so-called "melscale"). ${ }^{77}$ Contemporary music theorist John Rahn has stressed the dangers of

Reports in Ethnomusicology 2-3 (1987-88): 1-11, as well as the survey in [Jody Diamond], "Network," Balungan 5, no. 1 (1991): 34-36.

77 Roederer, Introduction to the Physics and Psychophysics of Music, 10. 
the so-called "numerological fallacy," which can be regarded as resulting from a conflation of a model (e.g., comprising numbers) and that which it models (e.g., perceived relations between tones and intervals). ${ }^{78}$ Nominalist philosopher Nelson Goodman not only has called into question the existence or reality of sets, in terms of which numbers have been defined within mathematics, but also has indicated ways in which such fictions can be replaced. ${ }^{79}$ In this tradition, the present study has focused on qualitative, partially quantitative, but essentially non-numerical, interpretations of tuning phenomena. ${ }^{80}$

Within unison- and octave-interaction, qualitative distinctions among undulation, impulse-beating, and roughness are acknowledged here as well as distinctions between faster and slower versions of these timbral effects. There are broad absolute differences in the physical correlates of the responses involved in such distinctions. These differences can be defined quite reliably in terms of bands of frequency-differences (e.g., ca. $7 \mathrm{cps}$ ) and seem to be relevant across cultures. Such apparent universals of human perception reflect aspects of physiology and/or neurology that can be assessed by psychoacoustics and appear to be inherited. Within such a biological framework, it would seem that various cultures specialize in various ways. For example, in Western concert music, beats seem to have been avoided, whereas in traditional Balinese music, unison- and octave-interaction have been systematized qualitatively and in a partially quantitative manner by way of relatively fixed increments or intervals of interaction-speed which perceptually match each other within certain spans of tolerance. Western concert music has featured tonal systems in which matching- and greater-than-relations, both functionally and merely intervallic, are coordinated with scale-degree sizes based on half-octave cycles. By contrast, Indonesian slendro has featured invariance at the level of half-octave cycling and a wide array of possible ways in which to realize mere and functional intervals.

Although the term "half-octave cycle" might seem to denote the sort of quantitative relation that Roederer specifies as relatively intractable for studies of perception, such cycles and the proportionate relations on which they are based can be defined entirely in terms of qualitative and partially quantitative relations, as well as the octave-interval. For example:

def $(x)(y)(z)(x+y M a P H x+z<=x+z A L A M L x+y, y+z)$.

Or in words: For any individuals (e.g., tones), $x, y$, and $z$, the sum of $x$ and $y$ is a major proportionate half of the sum of $x$ and $z$ if and only if $i$ ) the sum of $x$ and $z$ is at least as much larger than the sum of $x$ and $y$ [as the sum of $x$ and $y$ is] than the sum of $y$ and $z .^{81}$

78 John Rahn, Basic Atonal Theory (New York: Longman, 1980), 19-20.

79Nelson Goodman, The Structure of Appearance (Indianapolis: Bobbs-Merrill, 1966).

80Cf. Jay Rahn, "An Advance on A Theory for All Music: At-Least-As Predicates for Pitch, Time, and Loudness," Perspectives of New Music 30, no. 1 (1992): 158-83, esp. 168-69, and "A Non-Numerical Predicate of Wide Applicability for Perceived Intervallic Relations," Muzica 6, no. 2 (1995): 23-33.

81 See David Lewin, Musical Form and Transformation: 4 Analytic Essays (New Haven: Yale University Press, 1993), for a numerical formulation of the more restricted notion of "bisection," which corresponds to precise bisection, as distinguished from bisection in general (i.e., comprising approximate 
Minor proportionate halves, half-octaves, and scales can be defined readily, the latter comprising any group of tones such that (a) for each adjacent pair, $x$ and $y$, the lower, e.g., $x$, is a major proportionate half-octave below another tone in the scale, $z$, and the higher, e.g., $y$, a minor proportionate half-octave below $z$, and (b) for the adjacent pair, $z$ and $z^{\prime}$, of which $z$ is the lower, $y^{\prime}$, an octave above $y$, is related to $z$ and $z^{\prime}$ as $z$ is to $x$ and $y$, respectively. And higher-than, lower-than, and next-to-relations for pitch can be defined non-numerically in terms of the non-reflexive, non-symmetric, non-transitive predicate "is at least as high as." 82

Functional interval-relations depend for their definition on the octave, as do scale degrees. Scale degrees require as well the defined notion of a proportionate half which underlies not only the concept of disproportionateness, but also such other features of pitch collections as differences, ambiguities, contradictions, and incommensuracies. Not defined in terms of the octave, mere interval-relations account for the possibility of describing as wide or narrow any particular tone-pair that otherwise matches another pair of tones with regard to functional interval-sizes. ${ }^{83}$

Various rates of undulation, beating, and roughness can be formulated in a non-numerical manner parallel to pitch- and pitch-interval relations, the relevant perceptual dimensions being time and loudness. Not only can faster and slower rates be acknowledged non-numerically, but also increments of speed, that is, intervals of, or differences between, rates of undulation, beating, and roughness. Such a notion of speed increments has been couched within Balinese musical culture in terms of penjoregs and half-penjoregs. Timbral characterizations of these sorts can be applied not only to the unison but also to the octave. Both the unison and the octave are subject to absolute constraints that have been traced to physiology and neurology. Within such constraints, the octave appears to

bisection), both here and in, for example, Jay Rahn, "Some Recurrent Features of Scales," 45. Goodman, The Structure of Appearance, 50-51 provides a formulation of the notion of a sum of individuals, which, in a non-numerical, nominalist formulation, is, itself, an individual.

82E.g., along the lines of Rahn, "At-Least-As Predicates for Pitch, Time and Loudness," where "is inherently pitched," as distinguished from "is pitched," can be defined as follows: def (x)(IPx $\leftrightarrow x P R x)$, i.e., for any thing, $x, x$ is inherently pitched if and only if $x$ is pitch-related to $x$, and substituted for the predicate "is pitched," yielding all the theorems claimed in the earlier study that depend, for their proofs there, on the predicate "is pitched." Cf. also Jay Rahn, "From Similarity to Distance; From Simplicity to Complexity; From Pitches to Intervals; From Description to Causal Explanation," Music Theory Online 0 , no. 9 (1994).

83 The distinction between mere and functional interval-sizes can be conveyed by distinguishing between such statements as " $x$ is at least as large as $y$ " and " $x$ is, relative to $z$, at least as large as $y$," where $z$ satisfies the predicate "is an octave." A parallel formulation for time-intervals would convey the contrast between tempo giusto on the one hand and, on the other, rubato, accelerando, and ritardando, the temporal "octave" being a time-interval that is explicitly presented or implied on the basis of an explicitly presented time-interval. The sort of "counting" involved in comparing intervals in terms of scale-degrees can be framed along lines advanced by Goodman. The Structure of Appearance, 41. Goodman casts non-numerical versions of there being, for example, "three things" or "precisely two Poles [i.e., North and South]" entirely in logical terms, that is, employing no quantifiers beyond "there is at least one thing...such that," nor such non-nominalist ideas as that of a "set." Although he warns that his non-numerical formulation can be effective only "so long as time, space, and patience hold out," actual musical situations seem never to present the prospect of having to deal, on any occasion, with more than, for instance, seven such things as scale degrees within the arguably finite world of music. 
have provided a benchmark for relations that distinguish, around the world, functional and scalar pitch from mere pitch.

Whereas the formulations in preceding sections of this study are framed in terms of numbers, these are employed merely as a means to an end or tool for capturing what ultimately are non-numerical relations. In a converse manner, recent important studies by John Clough and his coworkers, which similarly comprise all music in their scope, define such qualitative and minimally quantitative aspects of scales as variety, multiplicity, and maximal evenness in terms of numbers and thereby illuminate, for example, the pre-quantitative, "ordinal" formulations of scales in ancient India. ${ }^{84}$ For studies of the present sort, one can proceed from non-numerical entities to numerical and back again fairly freely if one specifies sufficiently carefully correspondences between the two realms.

The mathematical relations involved in the normal distribution, which constitutes a foundation for the statistical measures employed here and in other tuning studies, might seem to be irreducibly numerical. However, as Rahn (1992) indicates, one can provide non-numerical substitutes for them by means of such logical operators as "and," "or," and "not," and such a non-numerical connective as "it is at least as probable that ... as it is that ...." As in recent attempts at replacing all of mathematics with non-numerical substitutes, ${ }^{85}$ there is no commitment, in the present, essentially nominalist formulation, to the "reality" of numbers, as such.

\section{Past and Future Studies}

The general, hierarchic framework described above provides a basis on which to assess previous studies of gamelan tuning. Conjectures concerning historical relations between Indonesian music and rather distant musical cultures ${ }^{86}$ and between slendro and pelog themselves ${ }^{87}$ seem not to have taken into account

84 John Clough and Gerald Myerson, "Variety and Multiplicity in Diatonic Systems," Journal of Music Theory 29 (1985): 249-70; John Clough and Jack Douthett, "Maximally Even Sets," and John Clough, Jack Douthett, N. Ramanathan, and Lewis Rowell, "Early Indian Heptatonic Scales and Recent Diatonic Theory," Music Theory Spectrum 15, no. 1 (1993): 36-58.

85E.g., Hartry Field, Science without Numbers: A Defence of Nominalism (Princeton, N.J.: Princeton University Press, 1980) and Geoffrey Hellman, Mathematics without Numbers: Towards a Modal-Structural Interpretation (Oxford: Clarendon Press, 1989), the former not without controversy.

86Erich Maria von Hornbostel, "Über ein akustisches Kriterium für Kulturzusammenhänge," Zeitschrift für Ethnologie 43 (1911): 601-15, and "Musikalische Tonsysteme," in Handbuch der Physik, ed. H. Geiger and K. Scheel (Berlin: Springer, 1927), 8: 425-29; Jaap Kunst, "A Musicological Argument for Cultural Relationship between Indonesia (Probably the Isle of Java) and Central Africa," Proceedings of the Musical Association 62 (1936): $57-76$ (also, in German, Anthropos 31 (1936): 131-40); Manfred Bukofzer, "Kann die 'Blasquintentheorie' zur Erklarung exotischer Tonsysteme beitragen?" Anthropos 32 (1937): 402-18, and "Blasquinte," in Musik in Geschichte und Gegenwart, ed. Friedrich Blume (Kassel u. Basel: Bärenreiter Verlag, 1949), 1: 1918-24; and A.M. Jones, "Indonesia and Africa: The Xylophone as a Culture-Indicator," African Music 2-3 (1961): 36-47, and Africa and Indonesia: The Evidence of the Xylophone and Other Musical and Cultural Factors (Leiden: E.J. Brill, 1964).

87 Manfred Bukofzer, "The Evolution of Javanese Tone-Systems," in Papers Read at the International Congress of Musicology Held at New York, Sept. 11-16, 1939, ed. Arthur Mendel, Gustave Reese, and Gilbert Chase (New York: Music Educators' National Conference for American Musicological Society, 1944), 241-50, and Jaap Kunst, Around von Hormbostel's Theory of the Cycle of Blown Fifths 
the enormous, albeit determinate, diversity among traditional Indonesian tunings in either of the two principal tone-systems. ${ }^{88}$ Such hypotheses have tended to be framed too precisely, or by contrast, have been so vague as to be, in principle, absolutely unfalsifiable. ${ }^{89}$ Needed instead is a medial theory of Indonesian tuning that makes room for the manifest variation among fixed-frequency ensembles and yet provides a framework in terms of which the characters or personalities of individual orchestras can be conveyed. The present, hierarchic formulation secures a possible basis for such a theory.

Within a hierarchic formulation of the present sort, A.M. Jones's descriptive thesis, whereby the tone "pelog" is consistently, i.e., in all registers, an errant tone around which other tones of the pelog system as a whole are tuned in a stable manner, ${ }^{90}$ could be tested determinately. Similarly, one might assess J. Murray Barbour's weak hypothesis concerning pelog, namely, that pelog consists of two categories of intervals, smaller and larger, and within these categories, intervals are "interchangeable"-original German "austauschbar" [= "match each other (?)"]. ${ }^{91}$

Of all studies that have attempted to deal with both slendro and pelog in a single perspective, Hood's 1966 article, "Sléndro and Pélog Redefined," seems to have stood the test of time best. Virtually every point in Hood's seminal, exploratory study has been vindicated. Similarly, Susilo's account of tuning personalities (in his review of the third edition of Kunst's classic study of Music in Java) has been borne out in the present report. In this investigation, I feel that Hood's and Susilo's observations are not only corroborated but also provided with an explanatory framework and systematic, precise means of assertion.

In particular, the individuality of specific ensembles, a notion stressed by both Hood and Susilo, is rendered precisely here in terms of amounts of dispersion and degrees of significance in the differences that emerge within a single orchestra (and also, one can presume reasonably, between ensembles). In particular, also, certain regularities of tuning (or "mis-tuning") are accounted for here in terms of well-established findings in the psychology of perception as well as Ohgushi's neurophysiological conjecture (i.e., with regard to the tendency for octaves to be stretched rather than compressed). What falls by the wayside in this process of description and explanation is such an essentially "agnostic" formulation as is advanced by Lentz. And given greater substance

(Amsterdam: Royal Institute for the Indies, Mededeling 76, 1948).

88 The equinonatonic slendro tuning outlined above would facilitate a single orchestra combining both pelog (e.g., along the lines of Rahn, "Javanese Pelog Tunings Reconsidered") and slendro (as broadly conceived here) with a minimum number of keys.

89E.g., Kathleen Schlesinger, The Greek Aulos: A Study of Its Mechanism and of Its Relation to the Modal System of Ancient Greek Music, Followed by a Survey of the Greek Harmoniai in Survival or Rebirth in Folk-Music (London: Methuen, 1938; repr. Groningen: Bouma's Boekhuis, 1970).

90A.M. Jones, "Towards an Assessment of the Javanese Pelog Scale," Ethnomusicology 7, no. 1 (1963): 22-25.

91 J. Murray Barbour, "Missverständnisse über die Stimmung des Javanischen Gamelans," Die Musikforschung 16 (1963): 315-23, esp. 320; cf., however, Rahn, "Javanese Pelog Tunings Reconsidered," 78. 
are the observations of Ornstein and Dowling as well as my own earlier hypotheses concerning scales in general and pelog in particular.

In the future, it would seem that the general, broad-gauge unity of slendro tunings might be framed in terms of the determinate, falsifiable, half-octave cycle formulation advanced above without endangering accounts of the individuality of specific ensembles. This end might be achieved by formulating particular tunings within the hierarchical framework outlined here. Within such a paradigm, a number of studies might be carried out. In particular, the tuning measurements recorded by Hood, Ornstein, and the Gadjah Mada group in Indonesia (comprising Surjodiningrat, Sudarjana and Susanto) are to be highly recommended as a basis for further analysis because of the evident care with which they have been reported. Throughout North America, there are quite a few Balinese gamelan angklung in particular and Indonesian gamelan in general. Detailed studies, including spectral analysis, could be made of these without incurring great expense (e.g., for travel and equipment). As well, analytic studies that try to link (a) the actual structures of traditional pieces for other, non-angklung ensembles to (b) the tunings of the instruments on which such pieces have been played-as is attempted here for angklung-would appear to be worthwhile. Finally, one might seek also to realize determinate pedagogies for developing world musicianship that would be based not only on reliable methods of training but also on well-established findings in music psychology and ethnomusicology, and on contemporary approaches to music theory.

\begin{abstract}
A detailed analysis of the spectra and frequencies of tones produced on metallophones in a Balinese orchestra clarifies a number of issues that arise in studying timbre and tuning. Among these are the relative importance of accuracy and completeness of measurements, the relevance of acoustical description to musical perception and style, the appropriateness of statistical generalizations and numerical formulations to musical practice and theory, and the possibility of bi-musical responses to divergent tuning systems. Byproducts of the investigation include a proposed basis for inter-cultural ear training and a novel formulation of the unison, octave, and other intervals (including wide and narrow variants), as well as traditional scales and interval collections that have existed side-by-side in widely varying forms within individual cultures.
\end{abstract}

